\title{
The Ricci tensor of almost parahermitian manifolds
}

\author{
Diego Conti and Federico A. Rossi
}

April 15, 2018

\begin{abstract}
We study the pseudoriemannian geometry of almost parahermitian manifolds, obtaining a formula for the Ricci tensor of the Levi-Civita connection. The formula uses the intrinsic torsion of an underlying $\operatorname{SL}(n, \mathbb{R})$ structure; we express it in terms of exterior derivatives of some appropriately defined differential forms.

As an application, we construct Einstein and Ricci-flat examples on Lie groups. We disprove the parakähler version of the Goldberg conjecture, and obtain the first compact examples of a non-flat, Ricci-flat nearly parakähler structure.

We study the paracomplex analogue of the first Chern class in complex geometry, which obstructs the existence of Ricci-flat parakähler metrics.
\end{abstract}

\section{Introduction}

Paracomplex geometry was introduced by Libermann [23] in analogy with complex geometry, it is defined by a tensor $K$ with $K^{2}=\mathrm{Id}$, whose eigenspaces are integrable distributions of dimension $n$. The local geometry is that of a product, but things become more complicated if a metric enters the picture. The natural compatibility condition to impose is that $K$ be an anti-isometry, so that $F=g(K \cdot, \cdot)$ defines a two-form; if this form is closed, the metric is said to be parakähler. Such a metric is necessarily of neutral signature; its holonomy is contained in $\operatorname{GL}(n, \mathbb{R})$, endowed with its standard action on $\mathbb{R}^{n} \oplus\left(\mathbb{R}^{n}\right)^{*}$.

Parakähler manifolds carry a natural bilagrangian structure; as such, they form a natural object of study in symplectic geometry (see [15, 20); they also provide a natural setting for the study of the mean curvature flow, which is proved to preserve Lagrangian submanifolds in [9], under some assumptions on the curvature. Parakähler geometry also finds applications in physics ([12) and in the study of optimal transport ([22]). We refer to [14] for a survey; a more recent reference is 2 .

Like in Kähler geometry, the Ricci tensor of a parakähler manifold is given by $-\frac{1}{2} d d^{c} \log |\phi|^{2}$, where $\phi$ is a local holomorphic volume form (see 20]); this indicates that the existence of a parallel paracomplex volume form forces the Ricci

MSC class: 53C15; 53C10, 53C29, 53C50.

Keywords: Einstein pseudoriemannian metrics, almost parahermitian structures, intrinsic torsion, Ricci tensor.

This work was partially supported by FIRB 2012 "Geometria differenziale e teoria geometrica delle funzioni" and GNSAGA of INdAM. 
to be zero. In terms of the restricted holonomy $\mathrm{Hol}_{0}$, this condition is equivalent to $\mathrm{Hol}_{0} \subset \mathrm{SL}(n, \mathbb{R})$; thus, pseudoriemaniann metrics of neutral signature with (restricted) holonomy contained in $\operatorname{SL}(n, \mathbb{R})$ are Ricci-flat.

This observation leads us to consider the smaller structure group $\operatorname{SL}(n, \mathbb{R})$ rather than $\operatorname{GL}(n, \mathbb{R})$; we do not impose integrability conditions, so that the eigendistributions of $K$ are not necessarily integrable, nor is $F$ necessarily closed. The failure of the holonomy condition is measured by a tensor called intrinsic torsion; it follows from the above that the intrinsic torsion determines the Ricci tensor. An explicit formula to this effect is the main result of this paper (Theorems 9, 11). We note that similar situations have been studied in [7] and [5] for the Riemannian holonomy groups $\mathrm{G}_{2}$ and $\mathrm{SU}(3)$; see also 24 for similar computations relative to the group $\operatorname{Sp}(n) \operatorname{Sp}(1)$. Our methods, however, are more akin to those of [10], and they allow us to find an explicit formula valid in any dimension.

Intrinsic torsion relative to the structure group $\operatorname{GL}(n, \mathbb{R})$ can be identified with the covariant derivative of $F$ under the Levi-Civita connection; the results of 17 imply that, for $n \geq 3, \mathrm{GL}(n, \mathbb{R})$-intrinsic torsion decomposes into eight components, corresponding to eight non-isomorphic $\mathrm{GL}(n, \mathbb{R})$-modules $W_{1} \oplus$ $\cdots \oplus W_{8}$. Under $\operatorname{SL}(n, \mathbb{R})$, these components remain irreducible, but two extra components appear, giving rise to ten intrinsic torsion classes. This situation is somewhat different from that of almost hermitian geometry, where in six dimensions the Gray-Hervella intrinsic torsion classes become reducible upon reducing from $\mathrm{U}(3)$ to $\mathrm{SU}(3)$ (see [19, 8]).

Beside the fundamental form $F$, a manifold with an $\mathrm{SL}(n, \mathbb{R})$-structure carries decomposable $n$-forms $\alpha, \beta$, characterized as volume forms on the two distributions defined by $K$. In fact, the structure group $\operatorname{SL}(n, \mathbb{R})$ is the largest subgroup of $\operatorname{GL}(2 n, \mathbb{R})$ that fixes the corresponding elements $F, \alpha$ and $\beta$ of $\Lambda\left(\mathbb{R}^{2 n}\right)$. These differential forms are closed if and only if the intrinsic torsion is zero (see Proposition 12); structure groups satisfying this condition are known as strongly admissible (see [6]). This allows us to restate our formula for the Ricci tensor purely in terms of exterior derivatives (Theorem 15).

The language of $\operatorname{SL}(n, \mathbb{R})$-structure enables us to construct a cohomological invariant of paracomplex manifolds, analogous to the first Chern class in complex geometry, which obstructs the existence of Ricci-flat parakähler metrics; we obtain a sufficient topological condition for its vanishing (Theorem 21). One difference with the Kähler case is that compact parakähler Einstein manifolds are necessarily Ricci-flat, as we show in Proposition 20. Thus, unlike in the compact Kähler case, the invariant does not quite describe whether compatible parakähler Einstein metrics have zero or non-zero scalar curvature. This is also not true in the non-compact setting, as we show by producing a non-compact parakähler Einstein manifold with nonzero scalar curvature where the invariant is zero (Example 22).

We then turn to the construction of Einstein examples. Examples of compact, Ricci-flat parakähler manifolds appear in [20; these examples, however, are also flat. Non-flat examples on compact nilmanifolds were constructed by the second author in 26; a similar example appears in Section 7. These examples are homogeneous, marking a difference between Riemannian and pseudoriemannian geometry: by [1, homogeneous Riemannian Ricci-flat manifolds are flat. Parakähler Einstein manifolds with non-zero scalar curvature that are homogeneous under a semisimple Lie group are classified in 2 . 
Outside the parakähler setting, one significant intrinsic torsion class consists of nearly parakähler structures, characterized by the fact that $\nabla K$ is skewsymmetric in the first two indices. In our language, this means that the $\operatorname{GL}(n, \mathbb{R})$ intrinsic torsion is contained in $W_{1} \oplus W_{5}$ at each point; we say that its intrinsic torsion class is $\mathcal{W}_{1}+\mathcal{W}_{5}$. Examples of Einstein and Ricci-flat nearly parakähler metrics are constructed in [21, [13] and [27]. In fact, it follows easily from our formula (see Corollary [16) that nearly parakähler manifolds of dimension six are automatically Einstein, as originally proved in 21.

As a more restrictive condition, we study the intrinsic torsion class $\mathcal{W}_{1}$; we show that this is Ricci-flat in all dimensions. Considering left-invariant structures on nilpotent Lie groups, we obtain several explicit examples in dimension eight (Theorem 32). These structures are automatically nearly parakähler and Ricci-flat; they are also non-flat, and the underlying manifold is compact. We note that the previously known examples of Ricci-flat nearly parakähler manifolds were either non-compact ([27]) or flat ([13]).

The intrinsic torsion class $\mathcal{W}_{2}$ is also Ricci-flat. This leads to a counterexample of the paracomplex version of the Goldberg conjecture as stated in 25, asserting that a compact, Einstein almost parakähler manifold is necessarily parakähler (Proposition 27). Notice that the Kähler version of the conjecture is known to hold for non-negative scalar curvature (29]); our example is Ricci-flat, showing that the paracomplex situation is different.

The intrinsic torsion class $\mathcal{W}_{3}$ is not Ricci-flat, but we are able to construct a compact Ricci-flat example on a nilmanifold. However, the class $\mathcal{W}_{4}$ is different: a nilpotent Lie group with an invariant structure with intrinsic torsion in $W_{4}+$ $W_{8}$ is necessarily parakähler (Proposition 35).

Observing that changing the sign of $K$ has the effect of swapping $W_{i}$ with $W_{i+4}$, this concludes the analysis of "pure" intrinsic torsion classes. By taking products, it follows that all intrinsic torsion classes that do not contain $\mathcal{W}_{4}$ or $\mathcal{W}_{8}$ can be realized as the intrinsic torsion class of a nilmanifold with a non-flat, Ricci-flat metric (Proposition 40).

\section{The structure group $\mathrm{GL}(n, \mathbb{R})$}

An almost paracomplex structure on a manifold of dimension $2 n$ is a decomposition of the tangent space in two subbundles of rank $n$. The tangent space is then modeled on a direct sum

$$
T=V \oplus H,
$$

where $V$ and $H$ are real vector spaces of dimension $n$; explicitly, we shall fix a basis $e_{1}, \ldots, e_{2 n}$ of $T$ with

$$
V=\operatorname{Span}\left\{e_{1}, \ldots, e_{n}\right\}, \quad H=\operatorname{Span}\left\{e_{n+1}, \ldots, e_{2 n}\right\},
$$

and denote by $e^{1}, \ldots, e^{2 n}$ the dual basis of $T^{*}$. In these terms, we can think of an almost paracomplex structure as a $\mathrm{GL}(V) \times \mathrm{GL}(H)$-structure. In analogy with complex geometry, one considers an endomorphism of $T$ with $K^{2}=$ Id, namely

$$
K=\operatorname{Id}_{V}-\operatorname{Id}_{H}=e^{i} \otimes e_{i}-e^{n+j} \otimes e_{n+j}
$$


here and in the sequel, summation over repeated indices is implied; we adopt the convention that lower case indices range from 1 to $n$, and upper case indices range from 1 to $2 n$.

It is clear that a manifold of dimension $2 n$ admits an almost paracomplex structure if and only if it admits a distribution of rank $n$; for example, $S^{2 n}$ does not have an almost paracomplex structure [30, Theorem 27.18]. Thus, the longstanding problem of whether the six-dimensional sphere admits an integrable complex structure has a trivial answer in the paracomplex setting.

Like in almost complex geometry, differential forms on an almost paracomplex structure can be decomposed according to type via

$$
\Lambda^{k} T^{*}=\bigoplus_{p+q=k} \Lambda^{p, q}, \quad \Lambda^{p, q}=\Lambda^{p} V^{*} \otimes \Lambda^{q} H^{*} ;
$$

in the literature, one also finds the notations $T M=T_{+} M \oplus T_{-} M$ and $\Lambda_{ \pm}^{p, q}$.

An almost paracomplex structure is said to be paracomplex or integrable if the two rank $n$ distributions are integrable. By the Frobenius Theorem, this is equivalent to requiring that the exterior derivative have the form

$$
d: \Lambda^{p, q} \rightarrow \Lambda^{p+1, q}+\Lambda^{p, q+1},
$$

or to the vanishing of the Nijenhuis tensor

$$
N(X, Y)=[X, Y]+[K X, K Y]-K[K X, Y]-K[X, K Y] .
$$

In the language of $G$-structures, integrability can be expressed in terms of intrinsic torsion. Recall that the intrinsic torsion of a $G$-structure takes values in the cokernel of the linear map $\partial_{G}$, defined as the restriction to $T^{*} \otimes \mathfrak{g}$ of the alternating map

$$
\partial: T^{*} \otimes \mathfrak{g l}(T) \rightarrow \Lambda^{2} T^{*} \otimes T, \quad e^{i} \otimes\left(e^{j} \otimes e_{k}\right) \rightarrow e^{i j} \otimes e_{k} ;
$$

more precisely, the intrinsic torsion is obtained by projecting on this space the torsion of any connection on the $G$-structure.

If $\partial_{G}$ has a left inverse $s$, any $G$-structure has a unique minimal connection, namely one with torsion in ker $s$. In the present case, the alternating map is not injective, but we still have the following:

Proposition 1. Every $\mathrm{GL}(V) \times \mathrm{GL}(H)$-structure admits a connection with torsion taking values in

$$
\Lambda^{2} V^{*} \otimes H+\Lambda^{2} H^{*} \otimes V
$$

the torsion $\Theta$ of any such connection is related to the Nijenhuis tensor via

$$
\Theta(X, Y)=-\frac{1}{4} N(X, Y) \text {. }
$$

Proof. The representation $\Lambda^{2} T^{*} \otimes T$ splits as

$$
\Lambda^{2,0} \otimes V+\Lambda^{2,0} \otimes H+\Lambda^{1,1} \otimes V+\Lambda^{1,1} \otimes H+\Lambda^{0,2} \otimes V+\Lambda^{0,2} \otimes H,
$$

and

$T^{*} \otimes(\mathfrak{g l}(V)+\mathfrak{g l}(H))=\left(S^{2} V^{*} \otimes V+S^{2} H^{*} \otimes H\right)+\left(\Lambda^{2,0} \otimes V+\Lambda^{0,2} \otimes H+\Lambda^{1,1} \otimes T\right)$, 
where the first component represents the kernel of the alternating map. It follows that the restriction of $\partial$ to the second component has a left inverse $s$ with kernel (2). Therefore, if $\bar{\omega}$ is any connection on the $\mathrm{GL}(V) \times \mathrm{GL}(H)$-structure with torsion $\bar{\Theta}$, the connection $\omega=\bar{\omega}-s(\bar{\Theta})$ has torsion $\bar{\Theta}-\partial(s(\bar{\Theta})) \in$ ker $s$. Applying the definition $\Theta(X, Y)=\nabla_{X} Y-\nabla_{Y} X-[X, Y]$, and using the fact that $V$ and $H$ are preserved by the connection $\omega$, we obtain the required relation.

Remark 1. In terms of an adapted coframe $e^{1}, \ldots, e^{2 n}$, the torsion of a connection such as in Proposition 1 may be written as

$$
\Theta=\left(d e^{i}\right)^{0,2} \otimes e_{i}+\left(d e^{n+i}\right)^{2,0} \otimes e_{n+i} .
$$

Given an almost paracomplex structure, a pseudoriemannian metric $g$ is called almost parahermitian if

$$
g(K X, K Y)=-g(X, Y) .
$$

Such a metric is necessarily of neutral signature $(n, n)$; relative to (1), it determines an isomorphism $V \cong H^{*}$. Accordingly, the structure group is reduced to $\mathrm{GL}(n, \mathbb{R})$, and the tangent space is modeled on the representation

$$
T=V \oplus V^{*} .
$$

Alternatively, we may think of an almost parahermitian structure as determined by a non-degenerate two-form $F$ of type $(1,1)$; form and metric are related via

$$
F(X, Y)=g(K X, Y) .
$$

In this context, the basis of $T$ can always be chosen so that

$$
g=e^{i} \odot e^{n+i}, \quad F=e^{1, n+1}+\cdots+e^{n, 2 n},
$$

where $e^{i} \odot e^{n+i}$ stands for $e^{i} \otimes e^{n+i}+e^{n+i} \otimes e^{i}$ and $e^{i, n+i}$ for $e^{i} \wedge e^{n+i}$; using the metric $g$, we will use the identification

$$
V^{*} \ni e^{i} \mapsto e_{n+i} \in H .
$$

Due to the existence of the volume form $F^{n}$, almost parahermitian manifolds are orientable.

Whilst a paracomplex manifold is always locally a product $M \times N$, one should not think of parahermitian geometry as a fancy way to describe Cartesian products. At the topological level, this can be seen from the following:

Proposition 2. Let $M$ and $N$ be manifolds of dimension n, and assume that $T M$ is not trivial. Then the product paracomplex structure on $M \times N$ does not admit a compatible parahermitian structure.

Proof. Let

$$
\pi_{1}: M \times N \rightarrow M, \quad \pi_{2}: M \times N \rightarrow N
$$

denote the projections. The $K$-eigenspaces for the product paracomplex structure on $M \times N$ are $\pi_{1}^{*} T M$ and $\pi_{2}^{*} T N$. If a compatible parahermitian structure exists, the vector bundles $\pi_{1}^{*} T M \cong\left(\pi_{2}^{*} T N\right)^{*}$ are isomorphic. Therefore, their restrictions to a submanifold $M \times\{y\}$ are also isomorphic. However, the restriction of $\pi_{1}^{*} T M$ is equivalent to $T M$, and the restriction of $\left(\pi_{2}^{*} T N\right)^{*}$ is trivial, which is absurd. 
At a point, we can think of the structure group $\operatorname{GL}(n, \mathbb{R})$ as the stabilizer of $F$ in $\mathrm{SO}(n, n)$. At the Lie algebra level, this amounts to setting $B$ and $C$ to zero in

$$
\mathfrak{s o}(n, n)=\left\{\left(\begin{array}{cc}
A & B \\
C & -{ }^{t} A
\end{array}\right) \mid B=-{ }^{t} B, C=-{ }^{t} C\right\} .
$$

Having a metric at our disposal, we can write orthogonal decompositions such as

$$
\mathfrak{s o}(n, n)=\mathfrak{g l}(n, \mathbb{R}) \oplus \mathfrak{g l}(n, \mathbb{R})^{\perp}=\mathfrak{s l}(n, \mathbb{R}) \oplus \mathfrak{s l}(n, \mathbb{R})^{\perp},
$$

where

$$
\mathfrak{s l}(n, \mathbb{R})=\{\operatorname{Tr}(A)=0, B=0=C\}, \quad \mathfrak{s l}(n, \mathbb{R})^{\perp}=\{A=\lambda I\} .
$$

It will be convenient to fix the isomorphism

$$
\Lambda^{2} T^{*} \ni \alpha \mapsto M_{\alpha} \in \mathfrak{s o}(n, n), \quad\left\langle M_{\alpha}(v), w\right\rangle=\alpha(v, w) .
$$

Explicitly,

$$
\begin{gathered}
e^{i j} \mapsto e^{i} \otimes e_{n+j}-e^{j} \otimes e_{n+i}, \quad e^{n+i, n+j} \mapsto e^{n+i} \otimes e_{j}-e^{n+j} \otimes e_{i}, \\
e^{i, n+j} \mapsto e^{i} \otimes e_{j}-e^{n+j} \otimes e_{n+i} .
\end{gathered}
$$

Lemma 3. Through the identification (3), the Lie bracket on $\mathfrak{s o}(n, n)$ satisfies

$$
\left[a_{i j} e^{i j}, b_{k l} e^{n+k, n+l}\right]=\frac{2}{n} a_{i j}\left(b_{i j}-b_{j i}\right) e^{k, n+k} \bmod \mathfrak{s l}(n, \mathbb{R})+\mathfrak{g l}(n, \mathbb{R})^{\perp} .
$$

Proof. Follows from

$$
\left[e^{i j}, e^{n+k, n+l}\right]=-\delta_{i l} e^{j, n+k}+\delta_{j l} e^{i, n+k}+\delta_{i k} e^{j, n+l}-\delta_{j k} e^{i, n+l} .
$$

An almost parahermitian structure is called parakähler if one (hence both) of $K$ and $F$ is parallel under the Levi-Civita connection. More generally, $\nabla F$ can be identified with the intrinsic torsion of a $\operatorname{GL}(n, \mathbb{R})$-structure; the latter is known to decompose into eight components 17.

All finite-dimensional irreducible representations of $\operatorname{GL}(n, \mathbb{R})$ appear inside some

$$
V^{\otimes^{r}} \otimes\left(V^{*}\right)^{\otimes^{s}},
$$

(see e.g. [16]). Relative to the Cartan subalgebra of diagonal matrices, let $L_{i}$ denote the weight that maps $\operatorname{diag}\left(a_{1}, \ldots, a_{n}\right)$ to $a_{i}$. For any $k+h \leq n$ and integers $\lambda_{1} \geq \ldots \geq \lambda_{k}, \lambda_{n} \geq \ldots \geq \lambda_{n-h}$, denote by $V_{\lambda_{1}, \ldots, \lambda_{k}}^{\lambda_{n}, \ldots, \lambda_{n-h}}$ the representation with highest weight

$$
\lambda_{1} L_{1}+\ldots+\lambda_{k} L_{k}-\left(\lambda_{n-h} L_{n-h}+\ldots+\lambda_{n} L_{n}\right) .
$$

These representations are also irreducible under $\operatorname{SL}(n, \mathbb{R})$, but notice that under $\operatorname{SL}(n, \mathbb{R})$ the numbers $\lambda_{1}, \ldots, \lambda_{n}$ are not determined uniquely by the representation.

With this notation, $V=V_{1}$ and $V^{*}=V^{1}$. More generally,

$$
\left(V_{\lambda_{1}, \ldots, \lambda_{k}}^{\lambda_{n}, \ldots, \lambda_{n-h}}\right)^{*}=V_{\lambda_{n}, \ldots, \lambda_{n-h}}^{\lambda_{1}, \ldots, \lambda_{k}} ;
$$


in addition we have

$$
\mathfrak{s l}(n, \mathbb{R})=V_{1}^{1}, \quad \Lambda^{k} V=\underbrace{V_{1, \ldots, 1}}_{k}, \quad S^{k} V=V_{k} .
$$

We shall say that a representation has type $(k, h)$ if it is the sum of irreducible representations of the form $V_{\lambda_{1}, \ldots, \lambda_{k}}^{\lambda_{n}, \ldots, \lambda_{n-h}}$. In terms of Young diagrams, this says that the rows from $k+1$ to $n-h-1$ have the same length. It is clear that $V^{\otimes^{r}}$ has type $(r, 0)$; dually, $\left(V^{*}\right)^{\otimes^{s}}$ has type $(0, s)$. It now follows easily from the Littlewood-Richardson rule that (4) has type $(r, s)$.

We can think of a representation of type $(r, s)$ as a representation of $\mathrm{GL}(n, \mathbb{R})$ for any choice of $n>r+s$. The decomposition into irreducible components is then independent of $n$. For instance, for $s \geq r$ we have

$$
\Lambda^{r} V \otimes \Lambda^{s} V^{*}=V_{\underbrace{1, \ldots, 1}_{r}}^{\overbrace{1, \ldots, 1}^{s}}+V_{\underbrace{\overbrace{1, \ldots, 1}^{1, \ldots, 1}}_{r-1}}^{s-1}+\cdots+V^{\overbrace{1, \ldots, 1}^{s-r}} ;
$$

this also holds for $r+s=n$. Equivalently, we can write

$$
\Lambda^{r, s}=\Lambda_{0}^{r, s}+\left\{F \wedge \sigma, \sigma \in \Lambda^{r-1, s-1}\right\} \cong \Lambda_{0}^{r, s}+\cdots+\Lambda_{0}^{1, s-r+1}+\Lambda^{0, s-r} .
$$

In terms of an appropriate map $\Lambda: \Lambda^{r, s} \rightarrow \Lambda^{r-1, s-1}$, we have

$$
\sigma=[\sigma]_{0}+F \wedge \Lambda(\sigma), \quad \sigma \in \Lambda^{r, s} .
$$

Explicitly,

$$
\left.\left.\Lambda(\gamma)=-\frac{1}{n-1} e_{i}\right\lrcorner e_{n+i}\right\lrcorner \gamma, \quad \gamma \in \Lambda^{2,1}+\Lambda^{1,2} .
$$

As a consequence of the Littlewood-Richardson rule, one obtains:

Proposition 4 (17]). The intrinsic torsion of a $\mathrm{GL}(n, \mathbb{R})$-structures lies in

$$
T^{*} \otimes \mathfrak{g l}(n, \mathbb{R})^{\perp}=\text { Coker } \partial_{\mathrm{GL}(n, \mathbb{R})} \cong W_{1}+\cdots+W_{8}
$$

where

$$
\begin{aligned}
& W_{1}=\Lambda^{3} V^{*}, \quad W_{2}=V^{2,1}, \quad W_{3}=V_{1,1}^{1}, \quad W_{4}=V, \\
& W_{5}=\Lambda^{3} V, \quad W_{6}=V_{2,1}, \quad W_{7}=V_{1}^{1,1}, \quad W_{8}=V^{*} .
\end{aligned}
$$

To compare with Proposition 1 observe that the $\mathrm{GL}(V) \times \mathrm{GL}(H)$-intrinsic torsion corresponds to $W_{1}+W_{2}+W_{5}+W_{6}$; however, this space only splits in two irreducible components under the enlarged structure group.

\section{$3 \quad \mathrm{SL}(n, \mathbb{R})$-structures}

In this section we turn to the structure group $\mathrm{SL}(n, \mathbb{R})$. The invariant elements of $\Lambda^{*} T$ under the action $\mathrm{SL}(n, \mathbb{R})$ are generated by

$$
F=e^{1, n+1}+\cdots+e^{n, 2 n}, \quad \alpha=e^{1, \ldots, n}, \quad \beta=e^{n+1, \ldots, 2 n} ;
$$

conversely, $\mathrm{SL}(n, \mathbb{R})$ is the largest group that fixes this subalgebra.

Given a $\mathrm{GL}(n, \mathbb{R})$-structure, a reduction to $\mathrm{SL}(n, \mathbb{R})$ is determined by a global, nowhere-vanishing form of type $(n, 0)$. Clearly, a $\mathrm{GL}(n, \mathbb{R})$-structure admits a reduction to $\mathrm{SL}(n, \mathbb{R})$ if and only if it admits a reduction to $\mathrm{GL}_{+}(n, \mathbb{R})$, i.e. the two rank $n$ distributions are orientable. 
Example 5. Consider the standard parahermitian structure on $\mathbb{R}^{4}=\mathbb{R}^{2} \times \mathbb{R}^{2}$. This structure is preserved by the group $\Gamma$ generated by the diffeomorphism

$$
(x, y, z, t) \mapsto(x+1,-y, z,-t) .
$$

Hence the quotient $\mathbb{R}^{4} / \Gamma$ has an induced parahermitian structure. In this case, the rank two subbundles are not orientable, and there is no global reduction to $\mathrm{SL}(n, \mathbb{R})$.

Remark 2. Given an almost parahermitian structure on $M$, namely a GL $(n, \mathbb{R})$ structure $P$, it is always possible to find a $2: 1$ cover of $M$ which admits a reduction to $\mathrm{SL}(n, \mathbb{R})$. Indeed, the quotient $P / \mathrm{GL}_{+}(n, \mathbb{R})$ is a $2: 1$ cover of $M$ which admits a tautological $\mathrm{GL}_{+}(n, \mathbb{R})$-structure.

The intrinsic torsion of a $\operatorname{SL}(n, \mathbb{R})$-structure takes values in the cokernel of the alternating map

$$
\partial_{\mathrm{SL}(n, \mathbb{R})}: T^{*} \otimes \mathfrak{s l}(n, \mathbb{R}) \rightarrow \Lambda^{2} T^{*} \otimes T ;
$$

since $\partial_{O(n, n)}$ is an isomorphism, we can identify this space with $T^{*} \otimes \mathfrak{s l}(n, \mathbb{R})^{\perp}$.

Proposition 6. The intrinsic torsion of an $\mathrm{SL}(n, \mathbb{R})$-structure lies in

$$
T^{*} \otimes \mathfrak{s l}(n, \mathbb{R})^{\perp}=T^{*} \otimes \mathfrak{g l}(n, \mathbb{R})^{\perp} \oplus W^{1,0} \oplus W^{0,1},
$$

where

$$
W^{1,0}=\partial\left(V^{*} \otimes \mathbb{R}\right) \cong V^{*}, \quad W^{0,1}=\partial(V \otimes \mathbb{R}) \cong V .
$$

The Levi-Civita connection can always be written in the form

$$
\omega^{L C}=\omega+\tau+\tilde{\lambda},
$$

with $\tau \in T^{*} \otimes \mathfrak{g l}(n, \mathbb{R})^{\perp}, \omega$ is an almost-parahermitian connection and

$$
\tilde{\lambda}=\lambda \otimes\left(\begin{array}{cc}
I & 0 \\
0 & -I
\end{array}\right),
$$

where $\lambda$ is a one-form. For future reference, we note that given a form $\sigma$,

$$
\partial(\tilde{\lambda})\lrcorner \sigma=\sum_{p, q}(p-q) \lambda \wedge \sigma^{p, q} .
$$

Thus, $\omega$ is the connection obtained from the Levi-Civita connection by projection on $\mathfrak{s l}(n, \mathbb{R})$; we shall refer to it as the minimal connection, and denote by $\nabla, D$ the corresponding covariant derivative and exterior covariant derivative. By construction, the torsion of $\omega$ is $\Theta=-\partial(\tau+\tilde{\lambda})$. The component $\tau$ can be decomposed as the sum of

$$
\tau=\tau_{1}+\cdots+\tau_{8},
$$

with each $\tau_{i}$ corresponding to a section of the bundle associated to $W_{i}$. Relative to the action of $\mathbb{R}^{*} \subset \mathrm{GL}(n, \mathbb{R})$, we can decompose $\tau$ into four components with weights $-3,1,3,-1$, namely

$$
\begin{gathered}
\tau_{1}+\tau_{2}=a_{i j k} e^{i} \otimes e^{j k} \in V^{*} \otimes \Lambda^{2} V^{*}, \quad \tau_{3}+\tau_{4}=c_{i j k} e^{i} \otimes e_{j k} \in V^{*} \otimes \Lambda^{2} V, \\
\tau_{5}+\tau_{6}=b_{i j k} e^{n+i} \otimes e_{j k} \in V \otimes \Lambda^{2} V, \quad \tau_{7}+\tau_{8}=d_{i j k} e^{n+i} \otimes e^{j k} \in V \otimes \Lambda^{2} V^{*} .
\end{gathered}
$$


Here, summation over all $i, j, k$ is implied, and we assume that $a_{i j k}=-a_{i k j}$.

The components $W_{4}, W_{8}, W^{1,0}$ and $W^{0,1}$ can be encoded in three one-forms

$$
f_{4}=a_{i} e^{n+i}, \quad f_{8}=b_{i} e^{i}, \quad \lambda=\lambda_{I} e^{I}
$$

characterized by

$$
\tau_{4}=a_{i}\left(e^{k} \otimes e_{k i}\right), \quad \tau_{8}=b_{i}\left(e^{n+k} \otimes e^{k i}\right), \quad \tilde{\lambda}=\lambda_{I} e^{I} \otimes\left(e^{k} \otimes e_{k}-e^{n+k} \otimes e_{n+k}\right) .
$$

A useful symmetry arises as follows. Given an $\operatorname{SL}(n, \mathbb{R})$-structure $P$, one can consider the $\operatorname{SL}(n, \mathbb{R})$-structure $P \sigma$, where $\sigma=\left(\begin{array}{cc}0 & I \\ I & 0\end{array}\right)$; this amounts to interchanging $V$ and $H$. An adapted coframe $e^{1}, \ldots, e^{2 n}$ for $P$ determines an adapted coframe

$$
e_{\sigma}^{1}, \ldots, e_{\sigma}^{2 n}=e^{n+1}, \ldots, e^{2 n}, e^{1}, \ldots, e^{n}
$$

relative to which the intrinsic torsion has the form

$$
\begin{gathered}
\left(a_{\sigma}\right)_{i j k}=b_{i j k}, \quad\left(b_{\sigma}\right)_{i j k}=a_{i j k}, \quad\left(c_{\sigma}\right)_{i j k}=d_{i j k}, \quad\left(d_{\sigma}\right)_{i j k}=c_{i j k}, \\
\left(\lambda_{\sigma}\right)_{i}=-\lambda_{n+i}, \quad\left(\lambda_{\sigma}\right)_{n+i}=-\lambda_{i}
\end{gathered}
$$

the minus sign originates from the action of $\sigma$ on $\left(\begin{array}{cc}I & 0 \\ 0 & -I\end{array}\right)$.

There are constraints on $\tau$ coming from the first Bianchi identity. Indeed, recall that given a tensorial $k$-form $\eta$, one has

$$
D \eta=\mathfrak{a}(\nabla \eta)+\Theta\lrcorner \eta,
$$

where $\Theta$ is the torsion and $\mathfrak{a}(\nabla \eta)=\left\langle\nabla \eta, \frac{1}{k !} \theta \wedge \cdots \wedge \theta\right\rangle$. In particular,

$$
D \Theta=D(-\partial(\tau+\tilde{\lambda}))=\mathfrak{a}(-\nabla \partial(\tau+\tilde{\lambda}))+\partial(\tau+\tilde{\lambda})\lrcorner \partial(\tau+\tilde{\lambda})
$$

must satisfy

$$
D \Theta=\Omega \wedge \theta \in \Lambda^{2} T^{*} \otimes \mathfrak{s l}(n, \mathbb{R}) \subset \Lambda^{3} T^{*} \otimes T .
$$

Define the equivariant maps

$$
\begin{array}{ll}
p: \Lambda^{3} T^{*} \otimes T \rightarrow \Lambda_{0}^{1,1}, & \eta \otimes v \mapsto[v\lrcorner \eta+(n-1) \Lambda \eta \wedge v\lrcorner F]_{\Lambda_{0}^{1,1}} ; \\
q: \Lambda^{3} T^{*} \otimes T \rightarrow \mathbb{R}, & \eta \otimes v \mapsto \Lambda(v\lrcorner \eta) .
\end{array}
$$

Proposition 7. The intrinsic torsion of an $\mathrm{SL}(n, \mathbb{R})$-structure satisfies

$$
\begin{gathered}
\left.\left.p\left(\mathfrak{a}\left(-\nabla \partial\left(\tau_{3}+\tau_{4}+\tau_{7}+\tau_{8}\right)\right)+\partial\left(\tau_{1}\right)\right\lrcorner \partial\left(\tau_{6}\right)+\partial\left(\tau_{2}\right)\right\lrcorner \partial\left(\tau_{5}\right)+\partial\left(\tau_{6}\right)\right\lrcorner \partial\left(\tau_{1}\right) \\
\left.\left.\left.+\partial\left(\tau_{5}\right)\right\lrcorner \partial\left(\tau_{2}\right)+\partial\left(\tau_{3}+\tau_{4}+\tau_{7}+\tau_{8}\right)\right\lrcorner \partial(\tilde{\lambda})\right)=0 \\
\left.q\left(\mathfrak{a}\left(-\nabla \partial\left(\tau_{4}+\tau_{8}\right)\right)+\partial\left(\tau_{4}+\tau_{8}\right)\right\lrcorner \partial(\tilde{\lambda})\right)=0 .
\end{gathered}
$$

Proof. As a first step, we prove that $p$ and $q$ kill $\Omega \wedge \theta$. Decomposing $\Lambda^{3} T^{*}$ under $\operatorname{GL}(n, \mathbb{R})$, one readily sees that $p$ and $q$ are only non-trivial on $\Lambda^{2,1} \otimes V+$ $\Lambda^{1,2} \otimes V^{*}$. Writing $\Omega=\Omega^{2,0}+\Omega^{1,1}+\Omega^{0,2}$, it follows that

$$
p(\Omega \wedge \theta)=p\left(\Omega^{1,1} \wedge \theta\right) .
$$


By linearity, we can assume that $\Omega^{1,1}$ has the form $e^{i, n+j} \otimes\left(e^{k} \otimes e_{h}-e^{n+h} \otimes e_{n+k}\right)$; using (5), we obtain

$$
\begin{aligned}
p\left(\Omega^{1,1} \wedge \theta\right) & =p\left(e^{i, n+j, k} \otimes e_{h}-e^{i, n+j, n+h} \otimes e_{n+k}\right) \\
= & {\left.\left.\left[\delta_{i h} e^{n+j, k}+e^{i, n+h} \delta_{k j}+e_{j}\right\lrcorner e^{i, k, n+h}+e^{n+i}\right\lrcorner e^{n+j, n+h, k}\right]_{\Lambda_{0}^{1,1}}=0 . }
\end{aligned}
$$

The Bianchi identity now implies

$$
0=p(\Omega \wedge \theta)=p(\mathfrak{a}(-\nabla \partial(\tau+\tilde{\lambda}))+\partial(\tau+\tilde{\lambda})\lrcorner \partial(\tau+\tilde{\lambda})),
$$

and the same holds for $q$.

From $\lambda \wedge \lambda=0$, we obtain $\partial(\tilde{\lambda})\lrcorner \partial(\tilde{\lambda})=0$; the component $\partial(\tilde{\lambda})\lrcorner \partial(\tau)$ gives no contribution because of Schur's lemma and (7). Similarly, $\nabla\left(\partial\left(\tau_{1}+\tau_{2}+\tau_{5}+\tau_{6}\right)\right)$ is in the kernel of $p$ and $q$ because it has no component in $\Lambda^{2,1} \otimes V+\Lambda^{1,2} \otimes V^{*}$.

Observe that

$$
\mathfrak{a}(\nabla \partial \tilde{\lambda})=d \lambda \wedge e^{k} \otimes e_{k}-d \lambda \wedge e^{n+k} \otimes e_{n+k},
$$

hence, using (5) again,

$$
\begin{aligned}
& p\left(\mathfrak{a}(\nabla \partial \tilde{\lambda})=\left[e_{k}\right\lrcorner\left(d \lambda \wedge e^{k}\right)-e_{n+k}\right\lrcorner\left(d \lambda \wedge e^{n+k}\right) \\
& \left.\left.+(n-1) \Lambda\left(d \lambda \wedge e^{k}\right) \wedge e^{n+k}+(n-1) \Lambda\left(d \lambda \wedge e^{n+k}\right) \wedge e^{k}\right)\right]_{\Lambda_{0}^{1,1}} \\
= & {\left.\left.\left.\left.\left[e_{k}\right\lrcorner\left(d \lambda \wedge e^{k}\right)-e_{n+k}\right\lrcorner\left(d \lambda \wedge e^{n+k}\right)-e_{k}\right\lrcorner\left(d \lambda \wedge e^{k}\right)+e_{n+k}\right\lrcorner\left(d \lambda \wedge e^{n+k}\right)\right]_{\Lambda_{0}^{1,1}}=0 ; }
\end{aligned}
$$

a similar calculation shows that $q(\mathfrak{a}(\nabla \partial \tilde{\lambda})=0$.

Writing

$$
\begin{aligned}
\left.\left.\partial\left(\tau_{3}+\tau_{4}\right)\right\lrcorner \partial\left(\tau_{7}+\tau_{8}\right)=4 c_{i j k} e^{i, n+j} \otimes e_{k}\right\lrcorner & \left(d_{h l m} e^{n+h, l} \otimes e_{n+m}\right) \\
& =-4 c_{i j k} d_{h k m} e^{i, n+j, n+h} \otimes e_{n+m},
\end{aligned}
$$

and symmetrically $\left.\partial\left(\tau_{7}+\tau_{8}\right)\right\lrcorner \partial\left(\tau_{3}+\tau_{4}\right)=-4 d_{i j k} c_{h k m} e^{n+i, j, h} \otimes e_{m}$, we find that

$$
\begin{aligned}
& \left.\left.p\left(\partial\left(\tau_{7}+\tau_{8}\right)\right\lrcorner \partial\left(\tau_{3}+\tau_{4}\right)+\partial\left(\tau_{7}+\tau_{8}\right)\right\lrcorner \partial\left(\tau_{3}+\tau_{4}\right)\right) \\
= & {\left[4 c_{i m k} d_{h k m} e^{i, n+h}-4 c_{i j k} d_{h k h} e^{i, n+j}+4 c_{i i k} d_{h k m} e^{n+h, m}-4 c_{i j k} d_{i k m} e^{n+j, m}\right.} \\
+ & \left.4 d_{i m k} c_{h k m} e^{n+i, h}-4 d_{i j k} c_{h k h} e^{n+i, j}+4 d_{i i k} c_{h k m} e^{h, n+m}-4 d_{i j k} c_{i k m} e^{j, n+m}\right]_{\Lambda_{0}^{1,1}}
\end{aligned}
$$

is zero, and the same for $q$.

Explicit computations shows that $p$ and $q$ annihilate $\left.\left.\partial\left(\tau_{2}\right)\right\lrcorner \partial\left(\tau_{6}\right)+\partial\left(\tau_{6}\right)\right\lrcorner \partial\left(\tau_{2}\right)$ and $\left.\left.\partial\left(\tau_{1}\right)\right\lrcorner \partial\left(\tau_{5}\right)+\partial\left(\tau_{5}\right)\right\lrcorner \partial\left(\tau_{1}\right)$.

Finally, observe that $\nabla \partial \tau_{i}$ lies in a module isomorphic to $T^{*} \otimes W^{i}$ which only contains a component isomorphic to $V_{1}^{1}$ for $i=3,4,7,8$, and to $\mathbb{R}$ for $i=4,8$. Similarly, $\left.\partial\left(\tau_{i}\right)\right\lrcorner \partial\left(\tau_{j}\right)$ lies in a module isomorphic to $W^{i} \otimes W^{j}$; the equivariance of $p$ and $q$ gives the statement.

Remark 3. The component $\tau$ of the intrinsic torsion depends only on the $\mathrm{GL}(n, \mathbb{R})$-structure; the component $\lambda$ only depends on the $\operatorname{SL}(n, \mathbb{R}) \times \operatorname{SL}(n, \mathbb{R})$ structure. The components $\tau_{1}, \tau_{2}, \tau_{5}, \tau_{6}$ depend on the paracomplex structure (see Proposition 1); more precisely, they are determined by

$$
\left(d e^{i}\right)^{0,2}, \quad\left(d e^{n+i}\right)^{2,0} .
$$




\section{Ricci curvature}

In this section we give a formula for the Ricci tensor of an $\mathrm{SL}(n, \mathbb{R})$-structure, expressed in terms of its intrinsic torsion. Even though the structure group is $\mathrm{SL}(n, \mathbb{R})$, all relevant representations have a natural action of $\mathrm{GL}(n, \mathbb{R})$, and the maps we consider in this section are $\mathrm{GL}(n, \mathbb{R})$-invariant; accordingly, we will regard two representations as isomorphic if they are under this larger group.

The basic idea is that, relative to the decomposition

$$
\Lambda^{2} T^{*} \otimes \mathfrak{s o}(n, n)=\Lambda^{2} T^{*} \otimes\left(\mathfrak{s l}(n, \mathbb{R}) \oplus \mathfrak{g l}(n, \mathbb{R})^{\perp} \oplus \mathbb{R}\right),
$$

the relevant part of the curvature of the Levi-Civita connection is determined by the last two components, which only depend on the intrinsic torsion. Indeed, writing the Levi-Civita connection form as in (6), its curvature decomposes as

$$
\Omega^{L C}=\left(\Omega+\frac{1}{2}[[\tau, \tau]]_{\mathfrak{s l}(n, \mathbb{R})}\right)+(D \tau+[\tilde{\lambda}, \tau])+\left(d \lambda-\frac{1}{n} F(\tau, \tau)\right) \otimes\left(\begin{array}{cc}
I & 0 \\
0 & -I
\end{array}\right),
$$

where we have used Lemma 3 . Here $D$ denotes the exterior covariant derivative of the minimal connection, and $F(\tau, \tau)$ denotes a 2-form obtained by contracting $\tau$ with itself using $F$. More precisely, let $\langle\cdot, \cdot\rangle$ denote the natural pairing between $V$ and $V^{*}$, and consider the skew bilinear form on $\Lambda^{2} V+\Lambda^{2} V^{*}$ such that

$$
\tilde{F}(\gamma, \sigma)=\langle\gamma, \sigma\rangle=-\tilde{F}(\sigma, \gamma), \gamma \in \Lambda^{2} V, \sigma \in \Lambda^{2} V^{*},
$$

and zero otherwise, and define

$$
F\left(\eta \otimes \gamma, \eta^{\prime} \otimes \gamma^{\prime}\right)=\tilde{F}\left(\gamma, \gamma^{\prime}\right) \eta \wedge \eta^{\prime} .
$$

Thus, for fixed $i, h, j \neq k$,

$$
F\left(e^{i} \otimes e_{j k}, e^{n+h} \otimes e_{n+j, n+k}\right)=e^{i} \wedge e^{n+h} .
$$

We shall also consider the 2 -form $\bar{F}(\tau, \tau)$ defined by

$$
\bar{F}\left(e^{I} \otimes e^{J} \otimes e^{K}, e^{H} \otimes e^{L} \otimes e^{M}\right)=-F\left(e^{I}, e^{H}\right) F\left(e^{J}, e^{L}\right) e^{K} \wedge e^{M} .
$$

We shall decompose the Ricci tensor of the Levi-Civita connection as

$$
\mathrm{Ric}=\operatorname{Ric}^{\prime}+\operatorname{Ric}^{\prime \prime}, \quad \operatorname{Ric}^{\prime} \in V^{*} \otimes V, \quad \operatorname{Ric}^{\prime \prime} \in S^{2} V \oplus S^{2} V^{*} .
$$

Here, $V^{*} \otimes V$ represents a subspace of $S^{2} T^{*}$, i.e. $e^{i} \otimes e^{n+j}$ stands for $e^{i} \odot e^{n+j}$. We will also identify this space with $\Lambda^{1,1}$ through

$$
V^{*} \otimes V \cong \Lambda^{1,1}, \quad e^{i} \otimes e^{n+j} \mapsto e^{i, n+j} .
$$

As a first approximation, the $V^{*} \otimes V$ part of the Ricci can be described as follows.

Lemma 8. The Ricci tensor of an $\operatorname{SL}(n, \mathbb{R})$-structure satisfies

$$
\operatorname{Ric}^{\prime}=2 \operatorname{Ric}^{\prime}(D \tau+[\tilde{\lambda}, \tau])+n(d \lambda)^{1,1}-F(\tau, \tau)^{1,1} .
$$


Proof. The Riemann tensor takes values in the kernel $\mathcal{R}$ of the skewing map

$$
S^{2}\left(\Lambda^{2} T^{*}\right) \rightarrow \Lambda^{4} T^{*}
$$

As a $\operatorname{GL}(n, \mathbb{R})$-module, $S^{2}\left(\Lambda^{2} T^{*}\right)$ decomposes as

$$
S^{2}\left(\Lambda^{2} T^{*}\right)=3 \mathbb{R}+3 V_{1}^{1}+2 V_{1,1}^{1,1}+V_{2}^{2}+U+U^{*},
$$

where $U=2 \Lambda^{2} V+\Lambda^{4} V+V_{2,2}+V_{1,1,1}^{1}+V_{2,1}^{1}+S^{2} V$. Since the Ricci contraction is equivariant, and $\operatorname{Ric}^{\prime}$ takes values in $V^{*} \otimes V=V_{1}^{1} \oplus \mathbb{R}$, we only need to consider the components of $\mathcal{R}$ isomorphic to $V_{1}^{1}$ and $\mathbb{R}$.

The three components of $S^{2}\left(\Lambda^{2} T^{*}\right)$ isomorphic to $V_{1}^{1}$ contain the highest weight vectors

$$
v_{1}=e^{n, k+n} \odot e^{k, n+1}, \quad v_{2}=e^{n, n+1} \odot e^{k, n+k}, \quad v_{3}=e^{n+1, k+n} \odot e^{k n},
$$

where $n v_{1}-2 v_{2}$ lies in $S^{2}(\mathfrak{s l}(n, \mathbb{R})), v_{2}$ in $\mathfrak{s l}(n, \mathbb{R})^{\perp} \otimes \mathfrak{s l}(n, \mathbb{R})$ and $v_{3}$ in $S^{2}\left(\mathfrak{s l}(n, \mathbb{R})^{\perp}\right)$. However $\mathcal{R}$ only contains two copies of $V_{1}^{1}$, generated by $v_{1}+v_{2}, v_{1}+v_{3}$.

Similarly, the components isomorphic to $\mathbb{R}$ in $S^{2}\left(\Lambda^{2} T^{*}\right)$ are generated by

$$
\begin{gathered}
w_{1}=e^{n+i, n+j} \otimes e^{i j}+e^{i j} \otimes e^{n+i, n+j} \in S^{2}\left(\mathfrak{g l}(n, \mathbb{R})^{\perp}\right), \\
w_{2}=e^{i, n+i} \otimes e^{j, n+j} \in S^{2}(\mathbb{R})
\end{gathered}
$$

and

$$
w_{3}-\frac{1}{n} w_{2} \in S^{2}(\mathfrak{s l}(n, \mathbb{R})), \quad w_{3}=\sum_{i, j} e^{j, n+i} \otimes e^{i, n+j} .
$$

The vectors $w_{1}+2 w_{2}, w_{2}+w_{3}$ generate the two copies of $\mathbb{R}$ in $\mathcal{R}$.

By equivariance, and neglecting components not isomorphic to $V_{1}^{1}$ and $\mathbb{R}$, which do not contribute to Ric $^{\prime}$, we may assume that the Riemann tensor has the form

$$
R=a\left(v_{1}+v_{2}\right)+b\left(v_{1}+v_{3}\right)+h\left(w_{1}+2 w_{2}\right)+k\left(w_{2}+w_{3}\right) .
$$

The Ricci contraction of the fixed generators is given by

$$
\begin{gathered}
\operatorname{Ric}\left(w_{1}\right)=-2(n-1) g, \quad \operatorname{Ric}\left(w_{2}\right)=g, \quad \operatorname{Ric}\left(w_{3}\right)=n g, \\
\operatorname{Ric}\left(v_{1}\right)=n e^{n} \odot e^{n+1}, \quad \operatorname{Ric}\left(v_{2}\right)=2 e^{n} \odot e^{n+1}, \quad \operatorname{Ric}\left(v_{3}\right)=(n-2) e^{n} \odot e^{n+1} ;
\end{gathered}
$$

with our choice of $R$, the Ricci tensor is

$$
\operatorname{Ric}^{\prime}=(a(n+2)+b(2 n-2)) e^{n} \odot e^{n+1}+(2(2-n) h+(1+n) k) g .
$$

Consider the projections

$$
\begin{gathered}
\pi_{\mathfrak{g l}^{\perp}}: \mathcal{R} \rightarrow \Lambda^{2} T^{*} \otimes \mathfrak{g l}(n, \mathbb{R})^{\perp}, \quad \pi_{\mathfrak{s l}}: \mathcal{R} \rightarrow \Lambda^{2} T^{*} \otimes \mathfrak{s l}(n, \mathbb{R}), \\
\pi_{\mathbb{R}}: \mathcal{R} \rightarrow \Lambda^{2} T^{*} \otimes \mathbb{R} .
\end{gathered}
$$

Since both the image of $\pi_{\mathfrak{g l}^{\perp}}$ and the image of $\pi_{\mathbb{R}}$ contain $V_{1}^{1} \oplus \mathbb{R}$, it is possible to recover Ric' by only considering these projections. Explicitly, we have

$$
D \tau+[\tilde{\lambda}, \tau]=\pi_{\mathfrak{g l}^{\perp}}(R), \quad\left(d \lambda-\frac{1}{n} F(\tau, \tau)\right) \otimes\left(\begin{array}{cc}
I & 0 \\
0 & -I
\end{array}\right)=\pi_{\mathbb{R}}(R) .
$$


Then

$$
\begin{gathered}
\pi_{\mathfrak{g}^{\perp}}(R)=b v_{3}+h w_{1}, \\
\pi_{\mathbb{R}}(R)=\left(2 h+k\left(1+\frac{1}{n}\right)\right) w_{2}+\left(a+\frac{2}{n}(a+b)\right) e^{n, n+1} \otimes\left(e^{k} \otimes e_{k}-e^{n+k} \otimes e_{n+k}\right) .
\end{gathered}
$$

Thus (14) gives

$$
\begin{gathered}
\operatorname{Ric}^{\prime}(D \tau+[\tilde{\lambda}, \tau])=b(n-2) e^{n} \odot e^{n+1}-2 h(n-1) g \\
\operatorname{Ric}^{\prime}\left(\left(d \lambda-\frac{1}{n} F(\tau, \tau)\right) \otimes\left(\begin{array}{cc}
I & 0 \\
0 & -I
\end{array}\right)\right)=\left(a+\frac{2}{n}(a+b)\right) e^{n} \odot e^{n+1}+\left(2 h+k\left(1+\frac{1}{n}\right)\right) g .
\end{gathered}
$$

It is now straightforward to verify that the linear combination

$$
\operatorname{Ric}^{\prime}=2 \operatorname{Ric}^{\prime}(D \tau+[\tilde{\lambda}, \tau])+n \operatorname{Ric}^{\prime}\left(D \tilde{\lambda}+\frac{1}{2}[[\tau, \tau]]_{\mathbb{R}}\right)
$$

is consistent with (13); the statement follows observing that for any two-form $\eta$, through the identification (12),

$$
\operatorname{Ric}^{\prime}\left(\eta \otimes\left(\begin{array}{cc}
I & 0 \\
0 & -I
\end{array}\right)\right)=\eta^{1,1} .
$$

It turns out that the explicit dependence on the minimal connection (i.e. the term $D \tau$ ) can be partly eliminated from the formula:

Theorem 9. The Ricci tensor of an $\mathrm{SL}(n, \mathbb{R})$-structure satisfies

$$
\begin{aligned}
& \operatorname{Ric}^{\prime}=2 \operatorname{Ric}^{\prime}\left(\mathfrak{a}\left(\nabla \tau_{3}\right)+\lambda^{1,0} \wedge \tau_{3}\right)+2(n-2) d f_{4}^{1,1}+n(d \lambda)^{1,1}-2(n-1) f_{4} \wedge f_{8} \\
& \quad+\left(2 n \Lambda\left(d f_{4}\right)-4(n-1)\left\langle f_{4}, f_{8}\right\rangle\right) F-10 F\left(\tau_{1}, \tau_{5}\right)+2 F\left(\tau_{1}, \tau_{6}\right)-4 F\left(\tau_{2}, \tau_{5}\right) \\
& \quad-2 F\left(\tau_{2}, \tau_{6}\right)+2 \bar{F}\left(\tau_{2}, \tau_{6}\right)-2 F\left(\tau_{3}, \tau_{7}+\tau_{8}\right)-2(n-1) F\left(\tau_{4}, \tau_{7}\right) .
\end{aligned}
$$

Proof. As $\left[\tilde{\lambda}, \tau_{i}\right]$ depends equivariantly on an element of $T^{*} \otimes W_{i}$, by equivariance, the only contribution of $[\tilde{\lambda}, \tau]$ to $\operatorname{Ric}^{\prime}$ comes from $\left[\tilde{\lambda}, \tau_{3}+\tau_{4}+\tau_{7}+\tau_{8}\right]$. Since $\tau_{3}+\tau_{4} \in V^{*} \otimes \Lambda^{2} V$ and $\tau_{7}+\tau_{8} \in V \otimes \Lambda^{2} V^{*}$,

$$
\operatorname{Ric}^{\prime}([\tilde{\lambda}, \tau])=\operatorname{Ric}^{\prime}\left(2 \lambda \wedge\left(\tau_{3}+\tau_{4}\right)-2 \lambda \wedge\left(\tau_{7}+\tau_{8}\right)\right) .
$$

By (11),

$$
\left.D\left(\tau_{1}+\tau_{2}\right)=-\partial(\tau+\tilde{\lambda})\right\lrcorner\left(\tau_{1}+\tau_{2}\right) \quad \bmod T^{*} \otimes\left(W_{1}+W_{2}\right) ;
$$

and the same holds for $\tau_{5}+\tau_{6}$; by equivariance,

$\left.\left.\operatorname{Ric}^{\prime}(D \tau)=\operatorname{Ric}^{\prime}\left(-\partial\left(\tau_{5}+\tau_{6}\right)\right\lrcorner\left(\tau_{1}+\tau_{2}\right)-\partial\left(\tau_{1}+\tau_{2}\right)\right\lrcorner\left(\tau_{5}+\tau_{6}\right)+D \tau_{3}+D \tau_{4}+D \tau_{7}+D \tau_{8}\right)$.

Since the Ricci tensor is symmetric, we can identify Ric' ${ }^{\prime}$ with its projection on $V^{*} \otimes V$; using Lemma 8

$\left.\operatorname{Ric}^{\prime}=2 \operatorname{Ric}^{\prime}\left(D \tau_{3}+D \tau_{4}-\partial\left(\tau_{1}+\tau_{2}\right)\right\lrcorner\left(\tau_{5}+\tau_{6}\right)+2 \lambda^{1,0} \wedge\left(\tau_{3}+\tau_{4}\right)\right)+n d \lambda^{1,1}-F(\tau, \tau)^{1,1}$.

Recalling that the contraction of $\partial\left(\tau_{7}+\tau_{8}\right)$ into $\tau_{3}+\tau_{4}$ is zero and (7), we obtain

$$
\begin{aligned}
\operatorname{Ric}^{\prime}\left(D \tau_{3}+D \tau_{4}\right) & \left.=\operatorname{Ric}^{\prime}\left(\mathfrak{a}\left(\nabla \tau_{3}+\nabla \tau_{4}\right)-\partial(\tilde{\lambda})\right\lrcorner\left(\tau_{3}+\tau_{4}\right)\right) \\
& =\operatorname{Ric}^{\prime}\left(\mathfrak{a}\left(\nabla \tau_{3}+\nabla \tau_{4}\right)-\lambda \wedge\left(\tau_{3}+\tau_{4}\right)\right) .
\end{aligned}
$$


Therefore,

$$
\begin{aligned}
\operatorname{Ric}^{\prime}=2 \operatorname{Ric}^{\prime}\left(\mathfrak{a}\left(\nabla \tau_{3}+\nabla \tau_{4}\right)-\partial\left(\tau_{1}+\tau_{2}\right)\right\lrcorner\left(\tau_{5}+\tau_{6}\right) & \left.+\lambda^{1,0} \wedge\left(\tau_{3}+\tau_{4}\right)\right) \\
& +n(d \lambda)^{1,1}-F(\tau, \tau)^{1,1} .
\end{aligned}
$$

Writing $\tau_{4}$ as in (10),

$$
\begin{aligned}
\operatorname{Ric}^{\prime}\left(2 \lambda^{1,0} \wedge \tau_{4}\right) & =2\left(\lambda_{i} a_{i} e^{k} \otimes e^{n+k}+(n-2) \lambda^{1,0} \otimes a_{i} e^{n+i}\right) \\
& =2(n-2) \lambda^{1,0} \otimes f_{4}+2\left\langle\lambda^{1,0}, f_{4}\right\rangle F .
\end{aligned}
$$

In order to rewrite the term containing the covariant derivative of $\tau_{4}$, we may assume $\nabla \tau_{4}=a_{i j} e^{j} \otimes\left(e^{k} \otimes e_{k i}\right)$; this implies

$$
\operatorname{Ric}\left(\mathfrak{a}\left(\nabla \tau_{4}\right)\right)=a_{i i} F+(n-2) a_{i j} e^{j} \otimes e^{n+i}=a_{i i} F+(n-2) \nabla f_{4} .
$$

On the other hand we have

$$
\begin{aligned}
\left(d f_{4}\right)^{1,1} & \left.=\mathfrak{a}\left(\nabla f_{4}\right)^{1,1}-\partial\left(\tau_{7}+\tau_{8}\right)\right\lrcorner f_{4}+\lambda^{1,0} \wedge f_{4} \\
& =a_{i j} e^{j, n+i}+2 d_{i j k} a_{k} e^{j, n+i}+\lambda^{1,0} \wedge f_{4} \\
& =a_{i j} e^{j, n+i}+F\left(\tau_{7}+\tau_{8}, \tau_{4}\right)+\lambda^{1,0} \wedge f_{4}
\end{aligned}
$$

where

$$
F\left(\tau_{8}, \tau_{4}\right)=\left\langle f_{4}, f_{8}\right\rangle F+f_{4} \wedge f_{8}
$$

In particular

$$
\Lambda\left(d f_{4}\right)=\frac{1}{n} a_{i i}+\left(1-\frac{1}{n}\right)\left\langle f_{4}, f_{8}\right\rangle+\frac{1}{n}\left\langle\lambda^{1,0}, f_{4}\right\rangle,
$$

giving

$$
\begin{aligned}
\operatorname{Ric}\left(\mathfrak{a}\left(\nabla \tau_{4}\right)\right)=\left(n \Lambda\left(d f_{4}\right)-(n-1)\left\langle f_{4}, f_{8}\right\rangle-\left\langle\lambda^{1,0}, f_{4}\right\rangle\right) F & \\
& +(n-2)\left(d f_{4}^{1,1}-F\left(\tau_{7}+\tau_{8}, \tau_{4}\right)-\lambda^{1,0} \wedge f_{4}\right) .
\end{aligned}
$$

Decomposing $\tau$ as in (8), we find

$$
\begin{aligned}
& F\left(\tau_{1}+\tau_{2}, \tau_{5}+\tau_{6}\right)=-2 a_{i j k} b_{h j k} e^{i} \wedge e^{n+h}, \\
& \bar{F}\left(\tau_{1}+\tau_{2}, \tau_{5}+\tau_{6}\right)=-4 a_{i j k} b_{i j h} e^{k} \wedge e^{n+h}
\end{aligned}
$$

then

$$
\begin{aligned}
\left.\operatorname{Ric}\left(\partial\left(\tau_{1}+\tau_{2}\right)\right\lrcorner\left(\tau_{5}+\tau_{6}\right)\right) & =4\left(a_{j i k} b_{k h j}-a_{i j k} b_{k h j}\right) e^{i} \otimes e^{n+h} \\
& =4 F\left(\tau_{1}, \tau_{5}\right)-2 F\left(\tau_{1}, \tau_{6}\right)+F\left(\tau_{2}, \tau_{5}\right)-\bar{F}\left(\tau_{2}, \tau_{6}\right) .
\end{aligned}
$$

By contrast,

$$
F(\tau, \tau)=2 F\left(\tau_{1}+\tau_{2}, \tau_{5}+\tau_{6}\right)+2 F\left(\tau_{3}+\tau_{4}, \tau_{7}+\tau_{8}\right) .
$$

Summing up,

$$
\begin{aligned}
& \operatorname{Ric}^{\prime}=2 \operatorname{Ric}^{\prime}\left(\mathfrak{a}\left(\nabla \tau_{3}\right)+\lambda^{1,0} \wedge \tau_{3}\right)+2(n-2)\left(d f_{4}-F\left(\tau_{7}+\tau_{8}, \tau_{4}\right)-\lambda^{1,0} \wedge f_{4}\right) \\
+ & 2\left(n \Lambda\left(d f_{4}\right)-(n-1)\left\langle f_{4}, f_{8}\right\rangle\right) F-8 F\left(\tau_{1}, \tau_{5}\right)+4 F\left(\tau_{1}, \tau_{6}\right)-2 F\left(\tau_{2}, \tau_{5}\right)+2 \bar{F}\left(\tau_{2}, \tau_{6}\right) \\
+ & 2(n-2) \lambda^{1,0} \wedge \tau_{4}+n(d \lambda)^{1,1}-2 F\left(\tau_{1}+\tau_{2}, \tau_{5}+\tau_{6}\right)-2 F\left(\tau_{3}+\tau_{4}, \tau_{7}+\tau_{8}\right),
\end{aligned}
$$

from which we obtain the statement. 
As an $\operatorname{SL}(n, \mathbb{R})$-module, $V^{*} \otimes V$ splits as the sum $V_{1}^{1} \oplus \mathbb{R}$; the two components of $\mathrm{Ric}^{\prime}$ in this decomposition can be written as $\operatorname{Ric}^{\prime}-s F$ and $s$, where $s$ denotes the scalar curvature

$$
s=\frac{1}{n} \operatorname{Ric}\left(e_{i}, e_{n+i}\right)
$$

Corollary 10. The scalar curvature of an $\mathrm{SL}(n, \mathbb{R})$-structure is given by

$$
\begin{aligned}
s=\frac{10}{n}\left\langle\tau_{1}, \tau_{5}\right\rangle-\frac{2}{n}\left\langle\tau_{2},\right. & \left.\tau_{6}\right\rangle-\frac{2}{n}\left\langle\tau_{3}, \tau_{7}\right\rangle \\
& +4(n-1) \Lambda d f_{4}-\frac{2(n-1)(2 n-1)}{n}\left\langle f_{4}, f_{8}\right\rangle+n \Lambda(d \lambda) .
\end{aligned}
$$

Proof. By construction, $s=\Lambda \mathrm{Ric}^{\prime}$; in addition, the components which do not contain a copy of $\mathbb{R}$ give no contribution to the scalar curvature by equivariance. This gives

$$
\begin{aligned}
s=(4 n-4) \Lambda d f_{4}-10 \Lambda F\left(\tau_{1}, \tau_{5}\right)-2 \Lambda F\left(\tau_{2}, \tau_{6}\right)+2 \Lambda \bar{F}\left(\tau_{2}, \tau_{6}\right) \\
+n \Lambda(d \lambda)-2 \Lambda F\left(\tau_{3}, \tau_{7}\right)-4(n-1)\left\langle f_{4}, f_{8}\right\rangle+2(n-1) \Lambda\left(f_{8} \wedge f_{4}\right) .
\end{aligned}
$$

A direct computation gives

$$
\begin{aligned}
& \Lambda\left(F\left(\tau_{1}+\tau_{2}, \tau_{5}+\tau_{6}\right)\right)=-\frac{1}{n}\left\langle\tau_{1}, \tau_{5}\right\rangle-\frac{1}{n}\left\langle\tau_{2}, \tau_{6}\right\rangle, \\
& \Lambda\left(\bar{F}\left(\tau_{1}+\tau_{2}, \tau_{5}+\tau_{6}\right)\right)=-\frac{2}{n}\left\langle\tau_{1}, \tau_{5}\right\rangle-\frac{2}{n}\left\langle\tau_{2}, \tau_{6}\right\rangle, \\
& \Lambda\left(F\left(\tau_{3}+\tau_{4}, \tau_{7}+\tau_{8}\right)\right)=\frac{1}{n}\left\langle\tau_{3}, \tau_{7}\right\rangle+\frac{1}{n}\left\langle\tau_{4}, \tau_{8}\right\rangle,
\end{aligned}
$$

proving the statement.

Remark 4. On a metric of neutral signature, the notion of "positive" scalar curvature is not meaningful: the pseudoriemannian metrics $g$ and $-g$ have the same Ricci tensor, but opposite scalar curvature. In our setup, this means that if we keep the splitting $V \oplus V^{*}$ but flip the sign of $F$, considering the $\operatorname{SL}(n, \mathbb{R})$ structure determined by the adapted coframe

$$
-e^{1}, \ldots,-e^{n}, e^{n+1}, \ldots, e^{2 n}
$$

then the $\tau$ and the $\lambda$ stay the same, but $s$ changes its sign.

The remaining part of the Ricci is given as follows. Denote by $\epsilon$ the symmetrization map

$$
\epsilon: T^{*} \otimes T^{*} \rightarrow S^{2} T^{*}, \quad \eta \otimes \gamma \mapsto \eta \odot \gamma .
$$

Theorem 11. The Ric" component of the Ricci tensor of an $\operatorname{SL}(n, \mathbb{R})$-structure satisfies

$$
\begin{aligned}
& {\left[\operatorname{Ric}^{\prime \prime}\right]_{S^{2} V}=\epsilon\left((n-1)\left(\nabla f_{4}-f_{4} \otimes f_{4}+\lambda^{0,1} \otimes f_{4}\right)\right.} \\
& \left.\left.\left.\left.\quad+\operatorname{Ric}\left(\mathfrak{a} \nabla \tau_{6}-\partial\left(\tau_{7}\right)\right\lrcorner\left(\tau_{5}+\tau_{6}\right)-\partial\left(\tau_{8}\right)\right\lrcorner \tau_{6}-\partial\left(\tau_{3}\right)\right\lrcorner \tau_{3}+3 \lambda^{1,0} \wedge \tau_{6}\right)^{0,2}\right), \\
& \quad\left[\operatorname{Ric}^{\prime \prime}\right]_{S^{2} V^{*}}=\epsilon\left((n-1)\left(\nabla f_{8}-f_{8} \otimes f_{8}-\lambda^{1,0} \otimes f_{8}\right)\right. \\
& \left.\left.\left.\left.\quad+\operatorname{Ric}\left(\mathfrak{a} \nabla \tau_{2}-\partial\left(\tau_{3}\right)\right\lrcorner\left(\tau_{1}+\tau_{2}\right)-\partial\left(\tau_{4}\right)\right\lrcorner \tau_{2}-\partial\left(\tau_{7}\right)\right\lrcorner \tau_{7}-3 \lambda^{0,1} \wedge \tau_{2}\right)^{2,0}\right) .
\end{aligned}
$$


Proof. In the case we consider the action of $\operatorname{GL}(n, \mathbb{R})$; two representations are isomorphic if they are under $\operatorname{SL}(n, \mathbb{R})$ and they have the same weight under $\mathbb{R}^{*}$.

The space $\Lambda^{2} T^{*} \otimes \Lambda^{2} T^{*}$ contains two copies of $S^{2} V$, contained in $\mathfrak{s l}(n, \mathbb{R}) \otimes$ $\mathfrak{s l}(n, \mathbb{R})^{\perp}$ and $\mathfrak{s l}(n, \mathbb{R})^{\perp} \otimes \mathfrak{s l}(n, \mathbb{R})$ respectively. Since the Riemann tensor is symmetric, this means that the $S^{2} V$ part of the Ricci is entirely determined by its component in $\mathfrak{s l}(n, \mathbb{R}) \otimes \mathfrak{s l}(n, \mathbb{R})^{\perp}$, i.e.

$$
[\operatorname{Ric}(\Omega)]_{S^{2} V}=\epsilon\left(\operatorname{Ric}\left([D \tau+[\tilde{\lambda}, \tau]]_{S^{2} V}\right)\right) .
$$

The only $W_{i} \otimes W_{j}$ that contain a copy of $S^{2} V$ are

$$
W_{5} \otimes W_{7}, W_{6} \otimes W_{7}, W_{6} \otimes W_{8}, W_{3} \otimes W_{3}, W_{4} \otimes W_{4} .
$$

Moreover, the two copies of $S^{2} V$ inside $T^{*} \otimes W_{i}$ are contained in $V \otimes W_{4}$ and $V^{*} \otimes W_{6}$. In consequence,

$$
\begin{aligned}
{[D \tau]_{S^{2} V}=} & {[\mathfrak{a}(\nabla \tau)-\partial(\tau)\lrcorner \tau-\partial(\tilde{\lambda})\lrcorner \tau]_{S^{2} V} } \\
= & {\left.\left[\mathfrak{a}\left(\nabla \tau_{4}+\nabla \tau_{6}\right)-\partial\left(\tau_{7}\right)\right\lrcorner\left(\tau_{5}+\tau_{6}\right)-\partial\left(\tau_{8}\right)\right\lrcorner \tau_{6} } \\
& \left.\left.\left.\quad-\partial\left(\tau_{3}\right)\right\lrcorner \tau_{3}-\partial\left(\tau_{4}\right)\right\lrcorner \tau_{4}-\lambda^{0,1} \wedge \tau_{4}+\lambda^{1,0} \wedge \tau_{6}\right]_{S^{2} V} .
\end{aligned}
$$

On the other hand (15) gives

$$
[[\tilde{\lambda}, \tau]]_{S^{2} V}=\left[2 \lambda^{0,1} \wedge \tau_{4}+2 \lambda^{1,0} \wedge \tau_{6}\right]_{S^{2} V}
$$

Now

$$
\left.\operatorname{Ric}\left(\partial\left(\tau_{4}\right)\right\lrcorner \tau_{4}\right)=(n-1) a_{i} a_{j} e^{n+i} \otimes e^{n+j}=(n-1) f_{4} \otimes f_{4} ;
$$

writing $a_{i j} e^{n+i} \otimes e^{n+j}$ for the $V \otimes V$ component of $\nabla f_{4}$, we obtain

$$
\operatorname{Ric}\left(\mathfrak{a} \nabla \tau_{4}\right)=(n-1) a_{i j} e^{n+i} \otimes e^{n+j} .
$$

Moreover

$$
\operatorname{Ric}\left(\lambda^{0,1} \wedge \tau_{4}\right)=(n-1) \lambda^{0,1} \otimes f_{4},
$$

giving the first formula in the statement. The second formula is obtained applying the symmetry $\sigma$ that interchanges $V$ and $H$.

\section{Forms}

In this section we find formulae that express the intrinsic torsion and the Ricci curvature in terms of exterior derivatives, rather than exterior covariant derivatives. In particular we relate the intrinsic torsion to the exterior derivatives of the forms $\alpha \in \Lambda^{n, 0}, \beta \in \Lambda^{0, n}, F \in \Lambda^{1,1}$.

First, we observe that $v \mapsto v \wedge \alpha, v \mapsto v \wedge \beta$ induce isomorphisms

$$
\pi_{0,1}: \Lambda^{n, 1} \rightarrow \Lambda^{0,1}, \quad \pi_{1,0}: \Lambda^{1, n} \rightarrow \Lambda^{1,0} .
$$

Proposition 12. The intrinsic torsion of an $\mathrm{SL}(n, \mathbb{R})$-structure determines $d F$, $d \alpha$ and $d \beta$ via

$$
\begin{gathered}
\left.\left.(d F)^{3,0}=-\partial\left(\tau_{1}\right)\right\lrcorner F, \quad(d F)^{2,1}=-\partial\left(\tau_{7}\right)\right\lrcorner F-2 f_{8} \wedge F, \\
\left.\left.(d F)^{0,3}=-\partial\left(\tau_{5}\right)\right\lrcorner F, \quad(d F)^{1,2}=-\partial\left(\tau_{3}\right)\right\lrcorner F-2 f_{4} \wedge F, \\
\left.(d \alpha)^{n, 1}=-\left(n \lambda^{0,1}+(n-1) f_{4}\right) \wedge \alpha, \quad(d \alpha)^{n-1,2}=-\partial\left(\tau_{5}+\tau_{6}\right)\right\lrcorner \alpha, \\
\left.(d \beta)^{1, n}=\left(n \lambda^{1,0}-(n-1) f_{8}\right) \wedge \beta, \quad(d \beta)^{2, n-1}=-\partial\left(\tau_{1}+\tau_{2}\right)\right\lrcorner \beta .
\end{gathered}
$$


Conversely,

$$
\begin{gathered}
f_{4}=-\frac{1}{2}(\Lambda(d F))^{0,1}, \quad f_{8}=-\frac{1}{2}(\Lambda(d F))^{1,0}, \\
\left.\left.\tau_{1}=\frac{1}{6} e^{i} \otimes e_{i}\right\lrcorner(d F)^{3,0}, \quad \tau_{5}=-\frac{1}{6} e^{n+i} \otimes e_{n+i}\right\lrcorner(d F)^{0,3}, \\
\left.\left.\tau_{2}=\frac{1}{2}\left\langle\left((d \beta)^{2, n-1}+\partial\left(\tau_{1}\right)\right\lrcorner \beta\right), e^{n+j, n+k} \wedge\left(e_{i}\right\lrcorner \alpha\right)\right\rangle e^{i} \otimes e^{j, k}, \\
\left.\left.\tau_{6}=\frac{1}{2}\left\langle\left((d \alpha)^{n-1,2}+\partial\left(\tau_{5}\right)\right\lrcorner \alpha\right), e^{j k} \wedge\left(e_{n+i}\right\lrcorner \beta\right)\right\rangle e^{n+i} \otimes e^{n+j, n+k}, \\
\left.\left.\tau_{3}=-\frac{1}{2} e^{i} \otimes e_{i}\right\lrcorner(d F)^{1,2}-\tau_{4}, \quad \tau_{7}=\frac{1}{2} e^{n+i} \otimes e_{n+i}\right\lrcorner(d F)^{2,1}-\tau_{8}, \\
\lambda^{0,1}=-\frac{1}{n} \pi_{0,1}(d \alpha)^{n, 1}-\frac{n-1}{n} f_{4}, \quad \lambda^{1,0}=\frac{1}{n} \pi_{1,0}(d \beta)^{1, n}+\frac{n-1}{n} f_{8} .
\end{gathered}
$$

Proof. The usual formula (11) gives

$$
\left.d \alpha=-\partial(\tau+\tilde{\lambda})\lrcorner \alpha=-\partial\left(\tau_{3}+\tau_{4}+\tau_{5}+\tau_{6}\right)\right\lrcorner \alpha-n \lambda^{0,1} \wedge \alpha .
$$

However, $\left.-\partial\left(\tau_{3}\right)\right\lrcorner \alpha$ is zero because it lies in $\Lambda^{n, 1} \cong V$ but $W_{3}$ is not isomorphic to $V$; in addition:

$$
\begin{gathered}
\left.\left.\partial\left(\tau_{4}\right)\right\lrcorner \alpha=-\sum_{i \neq k} a_{i} e^{k, n+i} \otimes e_{k}\right\lrcorner \alpha=\sum_{i \neq k} a_{i} e^{n+i} \wedge \alpha=(n-1) f_{4} \wedge \alpha \\
\left.d \alpha=-\partial\left(\tau_{5}+\tau_{6}\right)\right\lrcorner \alpha-\left(n \lambda^{0,1}+(n-1) f_{4}\right) \wedge \alpha
\end{gathered}
$$

where we have used (99). Similarly, $\left.\partial\left(\tau_{8}\right)\right\lrcorner \beta=(n-1) f_{8} \wedge \beta$,

$$
\left.d \beta=-\partial\left(\tau_{1}+\tau_{2}\right)\right\lrcorner \beta+\left(n \lambda^{1,0}-(n-1) f_{8}\right) \wedge \beta .
$$

For $F$ we compute

$$
\left.d F=-\partial(\tau+\tilde{\lambda})\lrcorner F=-2\left(f_{4}+f_{8}\right) \wedge F-\partial\left(\tau_{1}+\tau_{3}+\tau_{5}+\tau_{7}\right)\right\lrcorner F
$$

where we have used that $\left.\partial\left(\tau_{2}+\tau_{6}\right)\right\lrcorner F$ is zero because $W_{2}$ and $W_{6}$ are not isomorphic to any subspace of $\Lambda^{3}=\Lambda^{3,0} \oplus \Lambda^{2,1} \oplus \Lambda^{1,2} \oplus \Lambda^{0,3}$. By projecting this last formula on the different types of $(p, q)$-forms we get the statement.

We now prove the inverse formulae. From the last equation, we immediately get

$$
-\frac{1}{2} \Lambda(d F)=f_{4}+f_{8}
$$

and the equations for $\lambda^{1,0}$ and $\lambda^{0,1}$ are obvious.

If we set $\tau_{1}=e^{i} \otimes e^{j k}+e^{j} \otimes e^{k i}+e^{k} \otimes e^{i j}$; then

$$
\left.(d F)^{3,0}=-\partial\left(\tau_{1}\right)\right\lrcorner F=6 e^{i j k}
$$

and it follows that

$$
\left.\frac{1}{6} e^{h} \otimes e_{h}\right\lrcorner(d F)^{3,0}=\tau_{1} .
$$

Setting $\tau_{3}+\tau_{4}=e^{i} \otimes e^{n+j, n+k}$ we obtain

$$
\left.(d F)^{1,2}=-\partial\left(\tau_{3}+\tau_{4}\right)\right\lrcorner F=-2 e^{i, n+j, n+k},
$$


thus

$$
\left.\tau_{3}+\tau_{4}=-\frac{1}{2} e^{i} \otimes e_{i}\right\lrcorner(d F)^{1,2} .
$$

Finally if $\tau_{2}=a_{i j k} e^{i} \otimes e^{j k}$, for a fixed tensor $e^{n+h, n+l} \otimes e_{m}$ we have

$$
\begin{aligned}
\left\langle-\partial\left(\tau_{2}\right)\right\lrcorner & \left.\left.\beta, e^{n+h, n+l} \otimes e_{m}\right\lrcorner \alpha\right\rangle \\
& \left.\left.\left.=\left\langle-a_{i j k} e^{i j} \otimes e_{n+k}\right\lrcorner \beta+a_{i j k} e^{i k} \otimes e_{n+j}\right\lrcorner \beta, e^{n+h, n+l} \otimes e_{m}\right\lrcorner \alpha\right\rangle \\
& =-2 a_{h l m}+2 a_{l h m}=2 a_{m h l},
\end{aligned}
$$

since $a_{i j k}+a_{j k i}+a_{k i j}=0$ and $a_{i j k}=-a_{i k j}$. Thus,

$$
\left.\left.\tau_{2}=\frac{1}{2}\left\langle-\partial\left(\tau_{2}\right)\right\lrcorner \beta, e^{n+j, n+k} \wedge e_{i}\right\lrcorner \alpha\right\rangle e^{i} \otimes e^{j k} .
$$

The remaining equations are proved in the same way.

We can relate each component of the intrinsic torsion as we have done for $\tilde{\lambda}$, related to the 1 -form $\lambda$, and $\tau_{4}, \tau_{8}$ (see (9) ). Indeed, the component $\tau_{1}=$ $a_{i j k}\left(e^{i} \otimes e^{j k}+e^{j} \otimes e^{k i}+e^{k} \otimes e^{i j}\right)$ can be associated to the $(3,0)$-form $a_{i j k} e^{i j k}$, and analogously for $\tau_{5}$; we set

$$
\left.\left.f_{3}=-\partial\left(\tau_{3}\right)\right\lrcorner F, \quad f_{7}=-\partial\left(\tau_{7}\right)\right\lrcorner F .
$$

Finally, if $\tau_{2}=a_{i j k} e^{i} \otimes e_{n+j, n+k}$, we set

$$
\left.f_{2}=a_{i j k}\left(e_{n+j, n+k}\right\lrcorner \beta\right) \wedge e^{i} \in \Lambda^{1, n-2},
$$

and if $\tau_{6}=b_{i j k} e^{n+i} \otimes e_{j k}$ we set $\left.f_{6}=b_{i j k}\left(e_{j k}\right\lrcorner \alpha\right) \wedge e^{n+i} \in \Lambda^{n-2,1}$. We use the following convention: for any $p$-form $\sigma, p \geq 2$ and any bi-vector $e_{j k}$ the $(p-2)$-form $\left.e_{j k}\right\lrcorner \sigma$ is defined by

$$
\left.\left(e_{j k}\right\lrcorner \sigma\right)\left(X_{1}, \ldots, X_{p-2}\right)=\sigma\left(e_{j}, e_{k}, X_{1}, \ldots, X_{p-2}\right) ;
$$

equivalently, $\left.\left.\left.e_{j k}\right\lrcorner \sigma=e_{k}\right\lrcorner\left(e_{j}\right\lrcorner \sigma\right)$.

We can then restate the equations of the Ricci curvature by expressing the $\nabla \tau_{i}$ in terms of the exterior derivative of the forms $f_{i}$. To this purpose, we identify $V \otimes V$ with $\Lambda^{n-1,1}$ through

$$
v \otimes w \mapsto(v\lrcorner \alpha) \wedge w,
$$

enabling us to identify a subspace of $\Lambda^{n-1,1}$ isomorphic to $S^{2} V$. Similarly, to obtain a subspace isomorphic to $S^{2} V^{*}$ we identify $V^{*} \otimes V^{*}$ with $\Lambda^{1, n-1}$ via the isomorphism $v \otimes w \mapsto(v\lrcorner \beta) \wedge w$.

Lemma 13. The following equations hold for $f_{2}$ and $f_{6}$ :

$$
\begin{aligned}
{\left.\left[\operatorname{Ric}\left(\mathfrak{a}\left(\nabla \tau_{6}\right)-\partial\left(\tau_{7}+\tau_{8}\right)\right\lrcorner \tau_{6}\right)+3 \lambda^{1,0} \wedge \tau_{6}\right]_{S^{2} V} } & =\left[d f_{6}+n \lambda^{1,0} \wedge f_{6}\right]_{S^{2} V}, \\
{\left.\left[\operatorname{Ric}\left(\mathfrak{a}\left(\nabla \tau_{2}\right)-\partial\left(\tau_{3}+\tau_{4}\right)\right\lrcorner \tau_{2}\right)-3 \lambda^{0,1} \wedge \tau_{2}\right]_{S^{2} V^{*}} } & =\left[d f_{2}-n \lambda^{0,1} \wedge f_{2}\right]_{S^{2} V^{*}}
\end{aligned}
$$

and the following identities hold for $f_{4}$ and $f_{8}$ :

$$
\begin{aligned}
{\left.\left[\nabla f_{4}-f_{4} \otimes f_{4}+\lambda^{0,1} \otimes f_{4}\right]_{S^{2} V}=\left[(-1)^{n-1} d\left(f_{4}\right\lrcorner \alpha\right)\right]_{S^{2} V} } & \\
& +\frac{1}{2} n \lambda^{0,1} \odot f_{4}+(n-2) f_{4} \otimes f_{4}, \\
{\left.\left[\nabla f_{8}-f_{8} \otimes f_{8}-\lambda^{1,0} \otimes f_{8}\right]_{S^{2} V^{*}}=\left[(-1)^{n-1} d\left(f_{8}\right\lrcorner \beta\right)\right]_{S^{2} V^{*}} } & -\frac{1}{2} n \lambda^{1,0} \odot f_{8}+(n-2) f_{8} \otimes f_{8} .
\end{aligned}
$$


Proof. We prove the first equation. As usual, we start from the following:

$$
\begin{gathered}
\left.d f_{6}=\mathfrak{a}\left(\nabla f_{6}\right)-\partial(\tau+\tilde{\lambda})\right\lrcorner f_{6} . \\
\text { If } \nabla \tau_{6}=a_{i j k h} e^{i} \otimes e^{n+j} \otimes e_{k h}+b_{i j k h} e^{n+i} \otimes e^{n+j} \otimes e_{k h}, \text { then } \\
\left.\left.\nabla f_{6}=a_{i j k h} e^{i} \otimes\left(e_{k h}\right\lrcorner \alpha\right) \wedge e^{n+j}+b_{i j k h} e^{n+i} \otimes\left(e_{k h}\right\lrcorner \alpha\right) \wedge e^{n+j} .
\end{gathered}
$$

We are interested in the $(n-1,1)$-component of $d f_{6}$, and more precisely in the $S^{2}(V)$ part. We get:

$$
\left.\left[d f_{6}\right]^{n-1,1}=\left[\mathfrak{a}\left(\nabla f_{6}\right)-\partial\left(\tau_{7}+\tau_{8}+\tilde{\lambda}\right)\right\lrcorner f_{6}\right]^{n-1,1} .
$$

Therefore,

$$
\begin{aligned}
{\left[d f_{6}\right]_{S^{2} V} } & \left.\left.=\left[a_{i j k h} e^{i} \wedge\left(e_{k h}\right\lrcorner \alpha\right) \wedge e^{n+j}-(n-3) \lambda^{1,0} \wedge f_{6}-\partial\left(\tau_{7}+\tau_{8}\right)\right\lrcorner f_{6}\right]_{S^{2} V} \\
& \left.\left.=\left[2 a_{i j k i}\left(e_{k}\right\lrcorner \alpha\right) \wedge e^{n+j}-(n-3) \lambda^{1,0} \wedge f_{6}-\partial\left(\tau_{7}+\tau_{8}\right)\right\lrcorner f_{6}\right]_{S^{2} V} \\
& \left.=a_{i j k i} e^{n+k} \odot e^{n+j}-\left[(n-3) \lambda^{1,0} \wedge f_{6}+\partial\left(\tau_{7}+\tau_{8}\right)\right\lrcorner f_{6}\right]_{S^{2} V},
\end{aligned}
$$

where we have used the identification (16). On the other hand, we have:

$\operatorname{Ric}\left(\mathfrak{a}\left(\nabla \tau_{6}\right)\right)=\operatorname{Ric}\left(a_{i j k h} e^{i, n+j} \otimes\left(e^{n+k} \otimes e_{h}-e^{n+h} \otimes e_{k}\right)\right)=2 a_{i j k i} e^{n+j} \otimes e^{n+k}$.

Writing $\tau_{6}=b_{i j k} e^{n+i} \otimes e_{j k}$ and $\tau_{7}+\tau_{8}=d_{i j k} e^{n+i} \otimes e^{j k}$, we compute

$$
\left.\left[\operatorname{Ric}\left(-\partial\left(\tau_{7}+\tau_{8}\right)\right\lrcorner \tau_{6}\right)\right]_{S^{2} V}=2 b_{k h j} d_{i j k} e^{n+i} \odot e^{n+h} .
$$

Moreover,

$$
\left.\left.-\partial\left(\tau_{7}+\tau_{8}\right)\right\lrcorner f_{6}=4 b_{k h j} d_{i j k} e_{h}\right\lrcorner \alpha \wedge e^{n+i},
$$

and the $S^{2}(V)$ component is $2 b_{k h j} d_{i j k} e^{n+h} \odot e^{n+i}$. Finally, note that

$$
\left[\lambda^{1,0} \wedge f_{6}\right]_{S^{2} V}=\lambda_{k} b_{i j k} e^{n+j} \odot e^{n+i}=\left[\operatorname{Ric}\left(\lambda^{1,0} \wedge \tau_{6}\right)\right]_{S^{2} V},
$$

which concludes the proof of the first equation. The second one is proved in a similar way.

Consider the $(n-1,0)$-form $\left.\left.f_{4}\right\lrcorner \alpha=a_{i} e_{i}\right\lrcorner \alpha$. If $\nabla \tau_{4}=a_{i j} e^{n+i} \otimes e^{k} \otimes e_{k j}$ $+b_{i j} e^{i} \otimes e^{k} \otimes e_{k j}$ then

$$
\left.\left.\left.\nabla\left(f_{4}\right\lrcorner \alpha\right)=a_{i j} e^{n+i} \otimes e_{j}\right\lrcorner \alpha+b_{i j} e^{i} \otimes e_{j}\right\lrcorner \alpha .
$$

As usual, $\left.\left.\left.\left.d\left(f_{4}\right\lrcorner \alpha\right)=\mathfrak{a}\left(\nabla\left(f_{4}\right\lrcorner \alpha\right)\right)-\partial(\tau+\tilde{\lambda})\right\lrcorner\left(f_{4}\right\lrcorner \alpha\right)$, but we are interested in the $(n-1,1)$ part, and more precisely in the $S^{2}(V)$ component. We obtain:

$$
\begin{aligned}
{\left.\left[d\left(f_{4}\right\lrcorner \alpha\right)\right]_{S^{2}(V)} } & \left.\left.\left.\left.=\left[\mathfrak{a}\left(\nabla\left(f_{4}\right\lrcorner \alpha\right)\right)-\partial\left(\tau_{4}\right)\right\lrcorner\left(f_{4}\right\lrcorner \alpha\right)-(n-1) \lambda^{0,1} \wedge\left(f_{4}\right\lrcorner \alpha\right)\right]_{S^{2}(V)} \\
=(-1)^{n-1}\left[a_{i j} e_{j}\right\lrcorner \alpha & \left.\left.\left.\wedge e^{n+i}-(n-1) a_{j} e_{j}\right\lrcorner \alpha \wedge a_{i} e^{n+i}-(n-1) a_{j} e_{j}\right\lrcorner \alpha \wedge \lambda^{0,1}\right]_{S^{2}(V)} \\
& =(-1)^{n-1}\left[\nabla f_{4}-(n-1) f_{4} \otimes f_{4}-(n-1) f_{4} \otimes \lambda^{0,1}\right]_{S^{2}(V)} .
\end{aligned}
$$

This ends the proof of the equation involving $f_{4}$. A similar argument proves the equation for $f_{8}$. 
Lemma 14. The $V_{1}^{1}$-Ricci part of $\tau_{3}$ and $\tau_{7}$ can be related to $f_{3}$ and $f_{7}$ via the following equations:

$$
\begin{aligned}
& \left.\left[\operatorname{Ric}\left(\mathfrak{a}\left(\nabla \tau_{3}\right)+\lambda^{1,0} \wedge \tau_{3}\right)\right]_{V_{1}^{1}}=\frac{2-n}{2}\left[\Lambda\left(d f_{3}+\partial\left(\tau_{7}+\tau_{8}\right)\right\lrcorner f_{3}\right)\right]_{V_{1}^{1}}, \\
& \left.\left[\operatorname{Ric}\left(\mathfrak{a}\left(\nabla \tau_{7}\right)-\lambda^{0,1} \wedge \tau_{7}\right)\right]_{V_{1}^{1}}=\frac{2-n}{2}\left[\Lambda\left(d f_{7}+\partial\left(\tau_{3}+\tau_{4}\right)\right\lrcorner f_{7}\right)\right]_{V_{1}^{1}} .
\end{aligned}
$$

Proof. Let $h_{3}$ be the equivariant map $h_{3}: \Lambda_{0}^{1,1} \rightarrow T^{*} \otimes W_{3}, \quad e^{i, n+j} \mapsto \sum_{k} e^{k} \otimes e^{i} \otimes e^{n+k, n+j}-\frac{1}{n}\left(e^{i} \otimes e^{k} \otimes e^{n+k, n+j}\right)$, and suppose that $\nabla \tau_{3}=h_{3}\left(e^{n, n+1}\right)$; then

$$
\nabla f_{3}=2 e^{k} \otimes e^{n+k, n, n+1}+\frac{2}{n} e^{n} \otimes F \wedge e^{n+1} .
$$

Using (11) we have

$$
\begin{aligned}
d\left(f_{3}\right) & \left.=\mathfrak{a}\left(\nabla f_{3}\right)-\partial(\tau+\tilde{\lambda})\right\lrcorner f_{3} \\
& \left.=2 \frac{n+1}{n} F \wedge e^{n, n+1}-\partial(\tau)\right\lrcorner f_{3}+\lambda \wedge f_{3},
\end{aligned}
$$

and an easy computation shows:

$$
\left.\Lambda\left(d f_{3}+\partial\left(\tau_{7}+\tau_{8}\right)\right\lrcorner f_{3}-\lambda^{1,0} \wedge f_{3}\right)^{2,2}=2 \frac{n+1}{n} e^{n, n+1} .
$$

On the other hand we have:

$$
\operatorname{Ric}\left(\mathfrak{a}\left(\nabla \tau_{3}\right)\right)=\frac{(2-n)(n+1)}{n} e^{n} \otimes e^{n+1}
$$

thus,

$$
\left.\left[\operatorname{Ric}\left(\mathfrak{a}\left(\nabla \tau_{3}\right)\right)\right]_{V_{1}^{1}}=\frac{2-n}{2} \Lambda\left(d f_{3}+\partial\left(\tau_{7}+\tau_{8}\right)\right\lrcorner f_{3}-\lambda^{1,0} \wedge f_{3}\right)^{2,2} .
$$

By the same token, if $\lambda^{1,0} \otimes \tau_{3}=h_{3}\left(e^{n, n+1}\right)$, we see that

$$
\lambda^{1,0} \wedge f_{3}=2 \frac{n+1}{n} F \wedge e^{n, n+1},
$$

so

$$
\left[\operatorname{Ric}\left(\lambda^{1,0} \wedge \tau_{3}\right)\right]_{V_{1}^{1}}=\frac{(2-n)(n+1)}{n} \frac{n}{2(n+1)} \Lambda\left(\lambda^{1,0} \wedge f_{3}\right) .
$$

Summing up, we get

$$
\left.\left[\operatorname{Ric}\left(\mathfrak{a}\left(\nabla \tau_{3}\right)+\lambda^{1,0} \wedge \tau_{3}\right)\right]_{V_{1}^{1}}=\frac{2-n}{2} \Lambda\left(d f_{3}+\partial\left(\tau_{7}+\tau_{8}\right)\right\lrcorner f_{3}\right)^{2,2},
$$

which proves the first of the two equations. By considering the equivariant map $h_{7}: \Lambda_{0}^{1,1} \rightarrow T^{*} \otimes W_{7}, \quad e^{i, n+j} \mapsto \sum_{k} e^{n+k} \otimes e^{n+i} \otimes e^{k, j}-\frac{1}{n}\left(e^{n+i} \otimes e^{n+k} \otimes e^{k, j}\right)$ and using a similar procedure, the other equation follows. 
Using Lemmas 13 and 14 we are able to restate Theorems 9 and 11, expressing the Ricci curvature of the Levi-Civita connection without using the covariant derivative $\nabla \tau$. Note that the projection of $\left.\left.\operatorname{Ric}\left(-\partial\left(\tau_{7}\right)\right\lrcorner \tau_{5}-\partial\left(\tau_{3}\right)\right\lrcorner \tau_{3}\right)$ on tensors of type $(2,0)$ is now redundant and we can drop it; the same happens for the $S^{2} V^{*}$ component of the Ricci tensor.

Theorem 15. The Ricci tensor of an $\operatorname{SL}(n, \mathbb{R})$-structure satisfies:

$$
\begin{gathered}
\left.\operatorname{Ric}^{\prime}=(2-n)\left[\Lambda\left(d f_{3}+\partial\left(\tau_{7}+\tau_{8}\right)\right\lrcorner f_{3}\right)\right]_{V_{1}^{1}}+2(n-2) d f_{4}^{1,1}-2(n-1) f_{4} \wedge f_{8} \\
+\left(2 n \Lambda\left(d f_{4}\right)-4(n-1)\left\langle f_{4}, f_{8}\right\rangle\right) F-10 F\left(\tau_{1}, \tau_{5}\right)+2 F\left(\tau_{1}, \tau_{6}\right)-4 F\left(\tau_{2}, \tau_{5}\right) \\
\quad-2 F\left(\tau_{2}, \tau_{6}\right)+2 \bar{F}\left(\tau_{2}, \tau_{6}\right)-2 F\left(\tau_{3}, \tau_{7}+\tau_{8}\right)-2(n-1) F\left(\tau_{4}, \tau_{7}\right)+n(d \lambda)^{1,1}, \\
{\left[\operatorname{Ric}^{\prime \prime}\right]_{S^{2} V}=\epsilon\left((n-1)\left(\left[(-1)^{n-1} d\left(f_{4}\right\lrcorner \alpha\right)\right]_{S^{2} V}+\frac{1}{2} n \lambda^{0,1} \odot f_{4}+(n-2) f_{4} \otimes f_{4}\right)} \\
\left.\left.\left.\quad+\left[d f_{6}+n \lambda^{1,0} \wedge f_{6}\right]_{S^{2} V}+\operatorname{Ric}\left(-\partial\left(\tau_{7}\right)\right\lrcorner \tau_{5}-\partial\left(\tau_{3}\right)\right\lrcorner \tau_{3}\right)\right), \\
{\left[\operatorname{Ric}^{\prime \prime}\right]_{S^{2} V^{*}}=\epsilon\left((n-1)\left(\left[(-1)^{n-1} d\left(f_{8}\right\lrcorner \beta\right)\right]_{S^{2} V^{*}}-\frac{1}{2} n \lambda^{1,0} \odot f_{8}+(n-2) f_{8} \otimes f_{8}\right)} \\
\left.\left.\left.\quad+\left[d f_{2}-n \lambda^{0,1} \wedge f_{2}\right]_{S^{2} V^{*}}+\operatorname{Ric}\left(-\partial\left(\tau_{3}\right)\right\lrcorner \tau_{1}-\partial\left(\tau_{7}\right)\right\lrcorner \tau_{7}\right)\right) .
\end{gathered}
$$

Remark 5. In the notation of [20], a $D$-valued $(n, 0)$-form has the form

$$
\Phi=(\bar{e} p+e q)(\bar{e} \alpha+e \beta)=\bar{e}(p \alpha)+e(q \beta) .
$$

Assume that the structure is paracomplex; then this is holomorphic if and only if it is closed; this is equivalent to

$$
d(\log p)^{0,1}=n \lambda^{0,1}+(n-1) f_{4}, \quad d(\log q)^{1,0}=-n \lambda^{1,0}+(n-1) f_{8} .
$$

By [20, in the parakähler case the Ricci form is given by

$$
\operatorname{Ric}=-\tau \partial \bar{\partial} \log |\Phi|^{2}=-\partial_{+} \partial_{-} \log |\Phi|^{2} .
$$

In our case, $|\Phi|^{2}=p q$, hence

$$
\begin{aligned}
-\partial_{+} \partial_{-} p q & \left.=-\partial_{+}\left(n \lambda^{0,1}-(n-1) f_{4}\right)\right)+\partial_{-}\left(-n \lambda^{1,0}+(n-1) f_{8}\right) \\
& =\left(-n d \lambda+(n-1)\left(-d f_{4}+d f_{8}\right)\right)^{1,1} .
\end{aligned}
$$

This is one component in our expression for the Ricci which corresponds to the curvature of the canonical bundle. In the parakähler case, i.e. $\tau=0$, the expressions coincide.

As an application, we recover a result of 21, asserting that six-dimensional nearly parakähler structures are Einstein. Recall that an almost parakähler manifold is called nearly parakähler if $\nabla K$ is skew-symmetric in the first two indices; in our language, this means that the intrinsic torsion lies in $W_{1}+W_{5}$ (see e.g. [17]). From the paracomplex point of view, this means that under some identification $V \cong H^{*}$, the two components of the Nijenhuis tensor lie in

$$
\Lambda^{3} V^{*}+\Lambda^{3} H^{*}
$$

i.e., the Nijenhuis tensor is totally skew-symmetric; this is the condition that ensures the existence of a connection with skew-symmetric torsion 21. With this 
definition, a parakähler manifold is nearly parakähler; we shall say a structure is strictly nearly parakähler if $\tau_{1} \neq 0$ at each point. It was shown in 28] that sixdimensional strictly nearly parakähler structures can be characterized in terms of differential forms and exterior derivatives. In our language, we obtain:

Corollary 16. On a connected six dimensional manifold $M$, an almost parahermitian structure with $\tau_{1} \neq 0$ is strictly nearly parakähler if and only if it has a reduction to $\mathrm{SL}(3, \mathbb{R})$ such that, up to rescaling by a constant, either

$$
d F=\alpha+\beta, \quad d(\alpha-\beta)=-\frac{1}{3} F^{2}
$$

or

$$
d F=\alpha, \quad d \beta=\frac{1}{6} F^{2} .
$$

The metric is Einstein with $s=-\frac{5}{18}$ in the former case, and Ricci-flat in the latter.

Proof. It is clear from Proposition 12 that the two situations correspond to the intrinsic torsion classes $W_{1}+W_{5}$ or $W_{1}$.

Conversely, if the intrinsic torsion lies in $W_{1}+W_{5}$, with $\tau_{1} \neq 0$, necessarily $d F$ has a component of type $(3,0)$; we can choose the reduction so that it equals $\alpha$. Then

$$
d F=\alpha+k \beta, \quad(d \beta)^{2,2}=\frac{1}{6} F^{2},
$$

for some function $k$. If $k$ is not zero at some point, we have

$$
(d \alpha)^{3,1}=0, \quad(d \alpha)^{2,2}=-\frac{k}{6} F^{2}, \quad(d \beta)^{1,3}=-d(\log k) \wedge \beta .
$$

This implies that $d \lambda=0$. Therefore, the metric is Einstein with curvature $-\frac{5 k}{18}$; up to rescaling we can assume $k \equiv 1$.

Otherwise $k$ is identically zero; this implies that

$$
0=d^{2} \beta=d\left(3 \lambda \wedge \beta+\frac{1}{6} F^{2}\right)=3 d \lambda \wedge \beta-\frac{1}{2} \lambda \wedge F^{2}
$$

since $\lambda$ has type $(1,0)$, this implies that $\lambda=0$. Hence, the metric is Ricciflat.

The six-dimensional case is also special because a nearly parakähler 6-manifold gives rise to a nearly parallel $\mathrm{G}_{2}^{*}$-structure on a suitable warped product ([1]).

\section{The Bott-Chern class}

Whilst the component $\tau$ of the intrinsic torsion depends only on the $\operatorname{GL}(n, \mathbb{R})$ structure, the component $\lambda$ only depends on the $\mathrm{SL}(n, \mathbb{R}) \times \mathrm{SL}(n, \mathbb{R})$-structure. In analogy with the complex case, it is possible to define an invariant that does not depend on a metric, playing the same role as the first Chern class.

Recall that the (para)Bott-Chern class cohomology spaces are defined as

$$
H_{B C}^{p, q}=\frac{\operatorname{ker} d: \Omega^{p, q} \rightarrow \Omega^{p+1, q} \oplus \Omega^{p, q+1}}{\operatorname{Im} d d^{c}: \Omega^{p-1, q-1} \rightarrow \Omega^{p, q}},
$$

where $d^{c}=K \circ d \circ K$. They are not finite-dimensional in general [3]. 
Proposition 17. Let $(M, K)$ be a paracomplex manifold that admits a reduction to $\operatorname{SL}(n, \mathbb{R})$, say $(F, \alpha, \beta)$. If $(\tilde{F}, \tilde{\alpha}, \tilde{\beta})$ is another reduction to $\operatorname{SL}(n, \mathbb{R})$ with

$$
\tilde{\alpha}=e^{n(h+k)} \alpha, \quad \tilde{\beta}=e^{n(h-k)} \beta,
$$

then its intrinsic torsion satisfies

$$
\tilde{\lambda}=\lambda+d^{c} h-d k .
$$

In particular, the Bott-Chern class $[d \lambda]$ only depends on $K$.

Proof. Straightforward from Proposition 12

$$
\begin{gathered}
-n \tilde{\lambda} \wedge \tilde{\alpha}=d(\tilde{\alpha})=n(d h+d k) \wedge \tilde{\alpha}-n \lambda^{0,1} \wedge \tilde{\alpha} \\
n \tilde{\lambda} \wedge \tilde{\beta}=d(\tilde{\beta})=n(d h-d k) \wedge \tilde{\beta}+n \lambda^{1,0} \wedge \tilde{\beta} .
\end{gathered}
$$

Proposition 18. Let $(M, K)$ be a paracomplex manifold which admits a reduction to $\mathrm{SL}(n, \mathbb{R})$. If $(M, K)$ admits a compatible Ricci-flat parakähler metric, then $[d \lambda]=0$ in $H_{B C}^{1,1}$.

Proof. Theorem [15]gives Ric ${ }^{\prime}=d \lambda$. Since $[d \lambda]$ only depends on $K$, if a Ricci-flat parakähler metric exists necessarily $d \lambda=0$.

We do not know whether an analogue of the Aubin-Yau theorem holds [4, 31: Proposition 17 only tells us what the volume of a potential Ricci-flat parakähler metric should be, but does not guarantee existence.

On the other hand, we have the following:

Proposition 19. Let $(F, \alpha, \beta)$ be a paracomplex $\operatorname{SL}(n, \mathbb{R})$-structure on $M$. Then $M$ admits a parakähler-Einstein metric with $s \neq 0$ compatible with the same paracomplex structure if and only if there exists a function $h$ such that

$$
\left(d \lambda+d d^{c} h\right)^{n}=e^{2 n h} F^{n} .
$$

Proof. Any compatible $\operatorname{SL}(n, \mathbb{R})$-structure has the form $(\tilde{F}, \tilde{\alpha}, \tilde{\beta})$, with

$$
\tilde{\alpha}=e^{n(h+k)} \alpha, \quad \tilde{\beta}=e^{n(h-k)} \beta .
$$

Since $k$ does not affect the metric, it is no loss of generality to assume $k=0$. Then from Proposition 12 we have:

$$
d \tilde{\lambda}=d \lambda+d d^{c} h .
$$

Set $\tilde{F}=d \tilde{\lambda}$; then $(\tilde{F}, \tilde{\alpha}, \tilde{\beta})$ defines an $\operatorname{SL}(n, \mathbb{R})$-structure if and only if

$$
\tilde{F}^{n}=e^{2 n h} F^{n} .
$$

If this condition holds, the structure is automatically parakähler and Einstein, because $\tilde{F}$ is closed and because of Theorem 9 .

By the same token, a compatible parakähler-Einstein metric necessarily satisfies $\tilde{F}=d \tilde{\lambda}$. 
This condition is the analogue of the Monge-Ampère equation in complex geometry. A striking difference is that the volume form is automatically exact; this means that it makes no sense to assume the manifold is compact. In fact, compact Einstein parakähler manifolds are necessarily Ricci-flat:

Proposition 20. If $M$ is a compact, parakähler manifold, then

$$
\int_{M} s F^{n}=0
$$

A left-invariant parakähler structure on a unimodular Lie group has $s=0$.

Proof. By Corollary [10, $d \lambda \wedge F^{n-1}=\frac{1}{n} s F^{n}$; this form is exact because $F$ is symplectic. If $M$ is compact, Stokes' theorem gives the statement; the same argument applies to the case of a unimodular Lie group, since there are no exact invariant volume forms.

Remark 6. More generally, this argument applies to balanced almost parakähler structures, i.e. those where $F^{n-1}$ is closed; then, in the case of an invariant structure on a unimodular Lie group, $\Lambda(d \lambda)=0$ and $\tau_{4}=0=\tau_{8}$. Therefore, the expression for the scalar curvature reduces to

$$
s=\frac{10}{n}\left\langle\tau_{1}, \tau_{5}\right\rangle-\frac{2}{n}\left\langle\tau_{2}, \tau_{6}\right\rangle-\frac{2}{n}\left\langle\tau_{3}, \tau_{7}\right\rangle
$$

Another difference with the (compact) complex case is that an Einstein parakähler manifold with $s \neq 0$ can have $[d \lambda]=0$; in fact, even $H_{B C}^{1,1}=0$ as in Example 22. On a paracomplex manifold $M$, decompose $d$ as $\partial_{+}+\partial_{-}$,

$$
\partial_{+}: \Omega^{p, q} \rightarrow \Omega^{p+1, q}, \quad \partial_{-}: \Omega^{p, q} \rightarrow \Omega^{p, q+1} .
$$

We can then define the (para)Dolbeault cohomology

$$
H_{+}^{p, q}=\frac{\operatorname{ker} \partial_{+}: \Omega^{p, q} \rightarrow \Omega^{p+1, q}}{\operatorname{Im} \partial_{+}: \Omega^{p-1, q} \rightarrow \Omega^{p, q}}, \quad H_{-}^{p, q}=\frac{\operatorname{ker} \partial_{-}: \Omega^{p, q} \rightarrow \Omega^{p, q+1}}{\operatorname{Im} \partial_{-}: \Omega^{p, q-1} \rightarrow \Omega^{p, q}}
$$

Theorem 21. Let $(M, K)$ be a paracomplex manifold with

$$
H^{1}(M)=0=H^{2}(M)=H_{+}^{1,0}(M)=H_{-}^{0,1}(M)
$$

Then $H_{B C}^{1,1}$ is zero.

Proof. Let $\mathcal{Z}^{1,1}$ be the sheaf of closed $(1,1)$-forms, and let $\mathcal{K}$ be the sheaf of $d d^{c}$-closed functions; we have a short exact sequence of sheaves

$$
0 \rightarrow \mathcal{K} \rightarrow \mathcal{A}^{0} \stackrel{d d^{c}}{\longrightarrow} \mathcal{Z}^{1,1} \rightarrow 0
$$

This gives

$$
0 \rightarrow H^{0}(\mathcal{K}) \rightarrow H^{0}\left(\mathcal{A}^{0}\right) \stackrel{d d^{c}}{\longrightarrow} H^{0}\left(\mathcal{Z}^{1,1}\right) \rightarrow H^{1}(\mathcal{K}) \rightarrow 0
$$

where we have used the fact that $\mathcal{A}^{0}$ is acyclic. Under the present assumptions, the $d d^{c}$ lemma is equivalent to $H^{1}(\mathcal{K})=0$. 
Now consider the sheaves

$$
\mathcal{Z}_{ \pm}^{p, q}=\operatorname{ker} \partial_{ \pm}: \mathcal{A}^{p, q} \rightarrow \mathcal{A}^{p+1+q}
$$

then the sequence

$$
0 \rightarrow \mathcal{Z}^{0} \rightarrow \mathcal{Z}_{+}^{0,0} \oplus \mathcal{Z}_{-}^{0,0} \rightarrow \mathcal{K} \rightarrow 0
$$

is exact. Indeed, assume the first and second de Rham cohomology of an open set $U$ is zero; then

$$
\gamma \in \mathcal{K}(U) \Longrightarrow d d^{c} \gamma=0 \Longrightarrow d^{c} \gamma=d f \Longrightarrow \partial_{+}(\gamma-f)=0, \partial_{-}(\gamma+f)=0
$$

thus $\gamma \in \mathcal{Z}_{+}^{0,0}(U) \oplus \mathcal{Z}_{-}^{0,0}(U)$.

Taking now the associated long exact sequence, and using the fact that $H^{1}(M)$ and $H^{2}(M)$ are zero, we deduce

$$
H^{1}(\mathcal{K})=H^{1}\left(\mathcal{Z}_{+}^{0,0}\right) \oplus H^{1}\left(\mathcal{Z}_{+}^{0,0}\right)
$$

Since a paracomplex manifold is locally a product, the following sequence is exact:

$$
0 \rightarrow \mathcal{Z}_{+}^{0,0} \rightarrow \mathcal{A}^{0} \stackrel{\partial_{+}}{\rightarrow} \mathcal{Z}_{+}^{1,0} \rightarrow 0,
$$

giving the exact sequence in cohomology

$$
H^{0}\left(\mathcal{A}^{0}\right) \stackrel{\partial_{+}}{\longrightarrow} H^{0}\left(\mathcal{Z}_{+}^{1,0}\right) \rightarrow H^{1}\left(\mathcal{Z}_{+}^{0,0}\right) \rightarrow 0 .
$$

This shows that $H^{1}\left(\mathcal{Z}_{+}^{0,0}\right)=H_{+}^{1,0}(M)=0$, and a similar result applies to the minus component. Thus, $H^{1}(\mathcal{K})=0$, which is what we had to prove.

Example 22. On the solvable Lie group $G=\mathrm{Aff}_{+}(\mathbb{R}) \times \mathrm{Aff}_{+}(\mathbb{R}) \times \mathrm{Aff}_{+}(\mathbb{R})$, consider a left-invariant basis of one-forms $e^{1}, \ldots, e^{6}$ such that

$$
d e^{1}=d e^{4}=e^{14}, \quad d e^{2}=d e^{5}=e^{25}, \quad d e^{3}=d e^{6}=e^{36} .
$$

We consider the $\mathrm{SL}(3, \mathbb{R})$-structure for which this is an adapted coframe; it is parakähler and Einstein with $s \neq 0$. This Lie group is diffeomorphic to $\mathbb{R}^{6}$; in addition, it is easy to check that the space of leaves for both foliations is smooth and diffeomorphic to $\mathbb{R}^{3}$, so that Theorem 21] applies and the Bott-Chern class $[d \lambda]$ is zero. Notice however that up to multiple, there is only one invariant closed $(1,1)$-form: if a compatible Ricci-flat parakähler metric exists, it cannot be invariant.

We conclude this section with a remark concerning the holonomy group of a parakähler manifold. By construction, this is a $\operatorname{subgroup~of~} \operatorname{GL}(n, \mathbb{R})$. If it is also contained in $\mathrm{GL}_{+}(n, \mathbb{R})$, the bundle $\Lambda^{n, 0}$ is trivial and it is possible to fix a reduction to $\operatorname{SL}(n, \mathbb{R})$. Ricci-flatness means that the restricted holonomy is $\mathrm{SL}(n, \mathbb{R})$; therefore, the full holonomy group has the form $G \times \mathrm{GL}(n, \mathbb{R})$, with $G$ a discrete subgroup of $\mathrm{GL}_{+}(n, \mathbb{R})$.

Example 23. Let $\lambda>0$ be a real constant and let

$$
\Omega=\left\{(x, y) \in \mathbb{R}^{n} \times \mathbb{R}^{n} \mid x_{1}>\frac{1}{1-\lambda}\right\} .
$$


Let $g$ be the affine transformation

$$
(x, y) \mapsto\left(\lambda x+e_{1}, \lambda^{-1} y\right) .
$$

The group generated by $g$ acts on $\Omega$ in a free, proper discontinuous way. Let $M=\Omega /\langle g\rangle$ be the quotient.

The flat parakähler structure

$$
F=d x_{1} \wedge d y_{1}+\cdots+d x_{n} \wedge d y_{n}, \quad h=\sum d x_{i} \odot d y_{i}
$$

passes onto the quotient. It is easy to see that

$$
\left\{\left(\lambda^{k} d x_{1}, \ldots, \lambda^{k} d x_{n}, \lambda^{-k} d y_{1}, \ldots, \lambda^{-k} d y_{n}\right) \mid k \in \mathbb{Z}\right\}
$$

defines a parallel $\mathbb{Z}$-structure on $\Omega$; this structure also passes onto the quotient. Moreover, taking parallel transport on $\Omega$ and observing that

$$
\pi_{* g p}\left(\frac{\partial}{\partial x_{i}}\right)=\lambda^{-1} \pi_{* p}\left(\frac{\partial}{\partial x_{i}}\right), \quad \pi_{* g p}\left(\frac{\partial}{\partial y_{i}}\right)=\lambda \pi_{* p}\left(\frac{\partial}{\partial y_{i}}\right),
$$

one sees that the holonomy of $M$ is precisely $\mathbb{Z}$.

\section{Einstein examples}

In this section we show some examples of Einstein metrics associated to $\operatorname{SL}(n, \mathbb{R})$ structures on Lie groups. With one exception (Example 25), the Lie groups we consider are nilpotent with rational structure constants; therefore, each admits a compact quotient which carries an induced $\mathrm{SL}(n, \mathbb{R})$-structure, also Einstein.

We will represent Lie groups by their structure constants; for instance the quadruplet $(0,0,0,12)$ represents a four-dimensional Lie group with a basis of left-invariant one-forms $e^{1}, e^{2}, e^{3}, e^{4}$ such that

$$
d e^{1}=0, d e^{2}=0, d e^{3}=0, d e^{4}=e^{12} ;
$$

the standard $\mathrm{SL}(2, \mathbb{R})$-structure on this Lie group is the one defined by the coframe $e^{1}, e^{2}, e^{3}, e^{4}$.

Example 24. A non-flat example of a parakähler Ricci-flat manifold is the standard $\mathrm{SL}(n, \mathbb{R})$-structure on the nilpotent Lie algebra $(24,0,0,0,0,35)$; in this case the curvature is $-e^{34} \otimes e^{34}$.

Example 25. There also exist parakähler Einstein Lie groups with non-zero scalar curvature: consider the one-parameter family of Lie algebras

$$
(14,25,36, t 14, t 25, t 36), \quad t \in \mathbb{R} ;
$$

notice that these are not nilpotent (in fact, not even unimodular). The standard $\mathrm{SL}(n, \mathbb{R})$-structure is Einstein, and Ricci-flat when $t=0$. In addition, the parakähler metric is unique once one fixes the paracomplex structure, if one requires it to be invariant. 
Example 26. The Lie group $\left(0,0,0,-\frac{1}{3} e^{23},-\frac{1}{3} e^{31},-\frac{1}{3} e^{12}\right)$ is flat and nearly parakähler; in this case, the minimal connection is the flat connection.

We can modify the example in such a way that the minimal connection is a non-trivial element in the subspace of $T^{*} \otimes \mathfrak{s l}(3, \mathbb{R})$ isomorphic to $V_{2}$; imposing $d^{2}=0$, we obtain the following Lie algebra:

$$
\begin{gathered}
d e^{1}=-2 \lambda_{3} e^{12}-2 e^{23}+2 e^{13} \lambda_{2} \\
d e^{2}=-2 \lambda_{3} e^{12} \lambda_{2}-2 e^{23} \lambda_{2}+2 e^{13} \lambda_{2}^{2} \\
d e^{3}=-2 \lambda_{3} e^{23}+2 \lambda_{3} e^{13} \lambda_{2}-2 \lambda_{3}^{2} e^{12} \\
d e^{4}=\left(e^{36}-e^{25}\right) \lambda_{3} \lambda_{2}-\lambda_{3} e^{24}-\frac{1}{3} e^{23}+e^{34} \lambda_{2}-\lambda_{3}^{2} e^{26}+e^{35} \lambda_{2}^{2} \\
d e^{5}=\frac{1}{3} e^{13}-e^{34}+\lambda_{3} e^{15} \lambda_{2}-e^{35} \lambda_{2}+\lambda_{3}^{2} e^{16}-\left(e^{36}-e^{14}\right) \lambda_{3} \\
d e^{6}=\lambda_{3} e^{26}-\frac{1}{3} e^{12}+e^{24}-\lambda_{3} e^{16} \lambda_{2}+\left(e^{25}-e^{14}\right) \lambda_{2}-e^{15} \lambda_{2}^{2} .
\end{gathered}
$$

The standard structure on this Lie algebra is again flat and nearly parakähler. A suitable change of basis, namely

$$
\left\{-e^{2}+e^{1} \lambda_{2}, e^{3}-\lambda_{3} e^{1},-\frac{1}{6} e^{1}+e^{4}+e^{5} \lambda_{2}+\lambda_{3} e^{6}, \frac{1}{3} e^{1}+e^{4}+e^{5} \lambda_{2}+\lambda_{3} e^{6},-e^{6},-e^{5}\right\},
$$

shows the Lie algebra to be nilpotent and isomorphic to $(0,0,0,12,14,24)$.

It follows from Theorem [15 that if the intrinsic torsion is purely in $W_{2}$, then the metric is Ricci flat. This leads us to a counterexample to the paracomplex version of the Goldberg conjecture of almost-Kähler geometry. This "para-Goldberg" conjecture, as stated in 25], asserts that any compact almost parakähler Einstein manifold is parakähler.

Proposition 27. The standard $\mathrm{SL}(2, \mathbb{R})$-structure on the nilmanifold $(0,0,0,12)$ defines a compact, almost parakähler Ricci-flat manifold which is not parakähler. Hence, the para-Goldberg conjecture does not hold.

Proof. One easily sees that $\lambda=0$ and the only component of the intrinsic torsion is $\tau_{2}$, implying Ricci-flatness (in fact, this metric is flat). The fact that $d e^{4}$ has a component in $\Lambda^{2,0}$ shows that the paracomplex structure is not integrable.

Example 28. Another example in the almost parakähler class $W_{2}+W_{6}$ is the nilpotent Lie algebra

$$
(0,0,46,0,12,0)
$$

with the standard $\mathrm{SL}(3, \mathbb{R})$-structure; its intrinsic torsion is

$$
\tau_{2}=e_{2} \otimes e^{12}, \quad \tau_{6}=e_{6} \otimes e^{46} .
$$

This metric is Ricci-flat, but the Levi-Civita curvature is $e^{26} \otimes e^{26}$.

Example 29. A Ricci-flat example with intrinsic torsion in $W_{3}$ is given by the standard $\operatorname{SL}(n, \mathbb{R})$-structure on the nilpotent Lie algebra $(0,0,0,0,0,45)$; this structure is almost parakähler and flat, with

$$
\tau=\frac{1}{2} e_{3} \otimes e^{45} \in W_{3} .
$$


A non-flat example is given by the standard $\operatorname{SL}(n, \mathbb{R})$-structure on the 8dimensional nilmanifold $(0,0,0,0,0,0,56,57)$; then

$$
\tau=\tau_{3}=\frac{1}{2} e_{3} \otimes e^{56}+\frac{1}{2} e_{4} \otimes e^{57}
$$

and the Ricci tensor is zero, but the curvature is $-\frac{1}{4} e^{56} \odot e^{45}$.

The nearly parakähler examples we have shown so far are flat, and the geometry of flat nearly parakähler structures is fully understood (see [13]). We now illustrate a systematic approach to construct Ricci-flat nearly parakähler metrics that are not flat. Our examples have intrinsic torsion in $W_{1}$; by Theorem 15 . this condition ensures Ricci-flatness.

The intrinsic torsion of an almost paracomplex structure can be identified with the Nijenhuis tensor, which has two components

$$
N^{H}+N^{V} \in \Lambda^{2} V^{*} \otimes H+\Lambda^{2} H^{*} \otimes V .
$$

Under the structure group $\mathrm{GL}(n, \mathbb{R})$, these two irreducible modules decompose respectively into $W_{1}+W_{2}$ and $W_{5}+W_{6}$; by Proposition 1 we can write

$$
N^{H}=4 \partial\left(\tau_{1}+\tau_{2}\right), \quad N^{V}=4 \partial\left(\tau_{5}+\tau_{6}\right) .
$$

The key observation is that $N^{H}$ can be identified with a linear bundle map that does not depend on the almost paracomplex structure $K$ but only on the 1-eigendistribution $\mathcal{V}$, namely

$$
\tilde{N}^{H}: \mathcal{V}^{\circ} \rightarrow \Lambda^{2} \mathcal{V}^{*}
$$

where $\mathcal{V}^{\circ}$ is the subbundle of $T^{*} M$ whose fibre at $p$ is the annihilator of $\mathcal{V}_{p}$

Proposition 30. Let $\mathfrak{g}$ be a Lie algebra of dimension $2 n$; let $V \subset \mathfrak{g}$ be a subspace of dimension $n$ such that:

1. $\tilde{N}^{H}$ has rank three;

2. the image $W=\operatorname{Im} \tilde{N}^{H}$ consists of simple forms, i.e. if $\eta \in W$ then $\eta \wedge \eta=0$

3. there is no $\sigma \in V^{*} \backslash\{0\}$ such that $\sigma \wedge \eta=0$ for all $\eta \in W$.

Then for any subalgebra $H \subset \mathfrak{g}$ complementary to $V$ there is a $\operatorname{GL}(n, \mathbb{R})$ structure compatible with the splitting $\mathfrak{g}=V \oplus H$ such that

$$
\tau_{2}=0=\tau_{5}=\tau_{6}
$$

Proof. Conditions 1 and 2 imply that $V^{*}$ is spanned by $e^{1}, \ldots, e^{n}$ such that $W$ is either of the form

$$
\operatorname{Span}\left\{e^{12}, e^{13}, e^{23}\right\}
$$

or

$$
\operatorname{Span}\left\{e^{14}, e^{24}, e^{34}\right\} ;
$$

the latter possibility is ruled out by Condition 3 . 
Having fixed the splitting $V \oplus H$, we can identify $V^{\mathrm{o}}$ with $H^{*}$; in particular, we can write

$$
\tilde{N}^{H}=\eta^{1} \otimes e^{23}+\eta^{2} \otimes e^{31}+\eta^{3} \otimes e^{12},
$$

where $\eta^{1}, \eta^{2}, \eta^{3}$ are linearly independent elements of $H^{* *}$. Let $e^{n+1}, \ldots, e^{2 n}$ be a basis of $H^{*}$ such that $\eta^{i}\left(e^{n+j}\right)=\delta_{i j}$; relative to the coframe $e^{1}, \ldots, e^{2 n}$, we can write

$$
N^{H}=e^{23} \otimes e_{n+1}+e^{31} \otimes e_{n+2}+e^{12} \otimes e_{n+3} ;
$$

thus, this coframe defines an $\mathrm{SL}(n, \mathbb{R})$-structure such that

$$
\tau_{1}+\tau_{2}=\frac{1}{8}\left(e^{1} \otimes e^{23}+e^{2} \otimes e^{31}+e^{3} \otimes e^{12}\right)
$$

i.e. $\tau_{2}=0$. The fact that $\tau_{5}=0=\tau_{6}$ follows from $H$ being integrable.

A similar result holds for distributions $\mathcal{V}$ and $\mathcal{H}$ on a manifold, if Conditions $1-3$ are assumed to hold at each point, but we will only consider the case of Lie groups. Then, the condition $\tau_{2}=0$ can be expressed as a linear equation in $F$ by using the following contraction:

$$
\left.c:\left(\Lambda^{2} V^{*} \otimes H\right) \otimes \Lambda^{2} T^{*} \rightarrow \Lambda^{2} V^{*} \otimes V^{*}, \quad(\eta \otimes h) \otimes F \mapsto \eta \otimes h\right\lrcorner F .
$$

Corollary 31. Let $\mathfrak{g}=V \oplus H$ be a splitting such as in Proposition 30, and assume that $d\left(\Lambda^{0, n}\right) \subset \Lambda^{2, n-1}$. Then any non-degenerate form $F \in \Lambda^{1,1}$ such that

$$
d F \in \Lambda^{3,0}, \quad c\left(N^{H}, F\right) \in \Lambda^{3,0}
$$

defines a Ricci-flat $\operatorname{GL}(n, \mathbb{R})$-structure with intrinsic torsion in $W_{1}$.

Proof. Fix a compatible reduction to $\operatorname{SL}(n, \mathbb{R})$; then Proposition 12 implies

$$
\lambda=0, \quad \tau_{3}=0=\tau_{4}=\tau_{7}=\tau_{8} .
$$

By Proposition 30 $\tau_{2}, \tau_{5}$ and $\tau_{6}$ vanish as well; thus the intrinsic torsion is in $W_{1}$, and by Theorem 15 the metric is Ricci-flat.

This gives us an effective recipe to find Ricci-flat nearly parakähler examples: for a given Lie algebra $\mathfrak{g}$ of dimension $2 n$, we seek a subspace $V \subset \mathfrak{g}$ of dimension $n$ that satisfies the conditions of Proposition 30 , and then a complementary integrable distribution $H$ such that $d\left(\Lambda^{0, n}\right) \subset \Lambda^{2, n-1}$. At this point it is only a matter of computing the space of forms of type $(1,1)$ that satisfy the linear equations (17), and verifying whether it contains a non-degenerate form. Implementing this strategy with a computer on Lie algebras of the form $\mathfrak{h} \oplus \mathbb{R}$, where $\mathfrak{h}$ ranges among 7 -dimensional nilpotent Lie algebras as classified by [18, gives the following:

Theorem 32. Each Lie algebra in Table 1 admits a family of non-flat, Ricci-flat nearly-parakähler structures with intrinsic torsion $\tau_{1} \neq 0$ and non-zero curvature, depending on parameters $\lambda, \mu \neq 0$ and $k$.

We emphasize that this is not a classification; whilst our program did not find any other example, we do not know whether there exist other 8-dimensional nilpotent Lie algebras with a strictly nearly parakähler structure which is Ricciflat but not flat. 
Table 1: Ricci-flat nearly-parakähler structures on nilpotent Lie algebras

\begin{tabular}{l|l}
$\mathfrak{h}$ & $\mathfrak{g}$ (in adapted coframe) \\
\hline $0,0,0, e^{12}, e^{23}+e^{14}, e^{24}, e^{15}-e^{34}$ & $0,0,-\lambda e^{18}, 0,-\mu e^{23}+\lambda e^{78}, \mu e^{13},-\mu e^{12}+\lambda \mu e^{38}, 0$ \\
$0,0,0, e^{12}, e^{23}+e^{14}, e^{24}+e^{13},-e^{34}+e^{15}$ & $0,0,-\lambda e^{18}, 0, \lambda e^{78}-\mu e^{23},-\lambda \mu e^{28}+\mu e^{13}, \lambda \mu e^{38}-\mu e^{12}, 0$ \\
$0,0, e^{12}, 0, e^{24}+e^{13}, e^{23}, e^{34}+e^{15}+e^{26}$ & $0,-\lambda e^{18}, 0,0, \mu e^{23}+e^{17}+\lambda e^{68}-k \lambda e^{18},-\mu e^{13}+\lambda \mu e^{28}, \mu e^{12}, 0$ \\
$0,0,0, e^{12}, e^{23}+e^{14},-e^{24}+e^{13},-e^{34}+e^{15}$ & $0,0,-\lambda e^{18}, 0,-\mu e^{23}+\lambda e^{78}, \mu e^{13}+\lambda \mu e^{28},-\mu e^{12}+\lambda \mu e^{38}, 0$ \\
$0,0, e^{12}, 0, e^{13}+e^{24}, e^{23},-e^{26}+e^{15}+e^{34}$ & $0,-\lambda e^{18}, 0,0,-e^{17}+\mu e^{23}+\lambda e^{68}+k \lambda e^{18}, \lambda \mu e^{28}-\mu e^{13}, \mu e^{12}, 0$
\end{tabular}

\section{Non-existence results}

The examples of Section 7 correspond to left-invariant structures on nilpotent Lie groups; in this section we give some non-existence results in the same context.

Lemma 33. Every left-invariant paracomplex $\mathrm{SL}(n, \mathbb{R})$-structure on a nilpotent Lie group satisfies

$$
\lambda=\frac{n-1}{n}\left(f_{8}-f_{4}\right) .
$$

Proof. Let $\mathfrak{g}$ be a nilpotent Lie algebra with a fixed $\mathrm{SL}(n, \mathbb{R})$-structure. For any $X \in \mathfrak{g}, \operatorname{ad} X$ is nilpotent; the induced action on $\Lambda \mathfrak{g}^{*}$ is also nilpotent.

By Proposition 12

$$
d \alpha=\left(-n \lambda-(n-1) f_{4}\right) \wedge \alpha
$$

whenever $X$ is in $H, \alpha$ is an eigenvector for the nilpotent endomorphism ad $X$ with eigenvalue

$$
\left(-n \lambda-(n-1) f_{4}\right)(X)
$$

it follows that

$$
n \lambda^{0,1}=-(n-1) f_{4} .
$$

The same argument applied to $\beta$ yields $n \lambda^{1,0}=(n-1) f_{8}$.

Theorem 15 immediately implies:

Corollary 34. Invariant parakähler structures on nilpotent Lie groups are Ricci-flat.

In contrast with the Riemannian case, Ricci-flat nilpotent Lie groups are not necessarily abelian; Section 7 contains several examples. Alongside the parakähler case (Example 24), we have constructed Ricci-flat examples with intrinsic torsion in $W_{1}, W_{2}$ and $W_{3}$; the absence of the class $W_{4}$ can be explained by the following:

Proposition 35. A nilpotent Lie group with an invariant, Einstein almost parakähler structure with intrinsic torsion in $W_{4}+W_{8}$ is only Einstein if it is parakähler, in which case it is Ricci-flat.

Proof. Fix a reduction to $\mathrm{SL}(n, \mathbb{R})$; by Lemma 33

$$
d \lambda=\frac{n-1}{n}\left(d f_{8}-d f_{4}\right) .
$$


On the other hand, Proposition 12 gives $d F=-2\left(f_{4}+f_{8}\right) \wedge F$; taking the exterior derivative, $0=-2\left(d f_{4}+d f_{8}\right) \wedge F$, and consequently $d f_{8}=-d f_{4}$. In addition, this two-form has type $(1,1)$ because the structure is paracomplex. Thus,

$$
d \lambda=-2 \frac{n-1}{n} d f_{4} .
$$

The formula for Ric $^{\prime}$ gives

$$
\operatorname{Ric}^{\prime}=2(n-2) d f_{4}+n d \lambda-2(n-1) f_{4} \wedge f_{8} \bmod F
$$

Thus, the metric is Einstein if there exists $k$ such that

$$
d f_{4}+(n-1) f_{4} \wedge f_{8}=k F
$$

taking the exterior derivative,

$$
\begin{aligned}
(n-1) d f_{4} \wedge f_{8} & +(n-1) f_{4} \wedge d f_{4}=-2 k\left(f_{4}+f_{8}\right) \wedge F \\
= & -2\left(f_{4}+f_{8}\right) \wedge\left(d f_{4}+(n-1) f_{4} \wedge f_{8}\right)=-2\left(f_{4}+f_{8}\right) \wedge d f_{4}
\end{aligned}
$$

this gives

$$
(n+1) d f_{4} \wedge\left(f_{4}+f_{8}\right)=0,
$$

and decomposing according to type

$$
d f_{4} \wedge f_{4}=0, \quad d f_{4} \wedge f_{8}=0 .
$$

Therefore,

$$
d f_{4} \in \operatorname{Span}\left\{f_{4} \wedge f_{8}\right\}
$$

this is only possible if $d f_{4}$ is zero; by (18) this implies $f_{4}=f_{8}=0$, which in turn gives $\lambda=0$.

Remark 7. Given an $\operatorname{SL}(n, \mathbb{R})$-structure on a manifold, it follows from Proposition 12 that the intrinsic torsion is contained in $W_{4}+W_{8}+W^{1,0}+W^{0,1}$ if and only if $N=0$ and $d F=\theta \wedge F$ for some 1-form $\theta$; such structures are known in the literature as locally conformally parakähler 17. The result of Proposition 35 can be rephrased by saying that an Einstein, left-invariant locally conformally parakähler structure on a nilpotent Lie group is parakähler.

\section{Composite intrinsic torsion classes}

In this section we give a simple construction to build almost parahermitian manifolds in a prescribed intrinsic torsion class using almost parahermitian manifolds of lower dimension as building blocks.

We shall denote by $T_{n}$ the $2 n$-dimensional GL( $\left.n, \mathbb{R}\right)$-module (1); let $W_{i}\left(T_{n}\right)$ denote the corresponding intrinsic torsion spaces. We will also use a subscript $n$ to denote the $2 n$-dimensional forms $F_{n}, \alpha_{n}$ and $\beta_{n}$.

We identify $T_{n} \oplus T_{m}$ with $T_{n+m}$ by the isomorphism that maps the basis

$$
\left\{e^{1}, \ldots, e^{n}, f^{1}, \ldots, f^{m}, e^{n+1}, \ldots, e^{2 n}, f^{m+1}, \ldots, f^{2 m}\right\}
$$

onto the basis

$$
\left\{E^{1}, \ldots, E^{2(m+n)}\right\}
$$


here, $\left\{e_{i}\right\},\left\{f_{i}\right\}$ and $\left\{E_{i}\right\}$ are the standard bases of $T_{n}, T_{m}$ and $T_{n+m}$ respectively. We obtain the following relations between the forms $F, \alpha$ and $\beta$ :

$$
F_{n+m}=F_{n}+F_{m}, \quad \alpha_{n+m}=\alpha_{n} \wedge \alpha_{m}, \quad \beta_{n+m}=\beta_{n} \wedge \beta_{m} .
$$

Moreover, we have the following lemma:

Lemma 36. The following relations hold:

$$
\begin{gathered}
W_{i}\left(T_{n}\right) \subset W_{i}\left(T_{n+m}\right) \quad \text { for } i \neq 4,8, \\
W_{4}\left(T_{n}\right) \subset W_{3}\left(T_{n+m}\right) \oplus W_{4}\left(T_{n+m}\right), \quad W_{8}\left(T_{n}\right) \subset W_{7}\left(T_{n+m}\right) \oplus W_{8}\left(T_{n+m}\right) .
\end{gathered}
$$

Moreover, the projections

$$
\begin{aligned}
& W_{3}\left(T_{n}\right) \oplus W_{4}\left(T_{n}\right) \oplus W_{3}\left(T_{m}\right) \oplus W_{4}\left(T_{m}\right) \rightarrow W_{3}\left(T_{n+m}\right) \\
& W_{7}\left(T_{n}\right) \oplus W_{8}\left(T_{n}\right) \oplus W_{7}\left(T_{m}\right) \oplus W_{8}\left(T_{m}\right) \rightarrow W_{7}\left(T_{n+m}\right)
\end{aligned}
$$

are injective.

Proof. Consider the commutative diagram

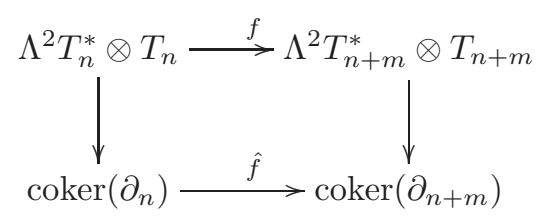

where $f\left(a_{I J K} e^{I J} \otimes e_{K}\right)=a_{I J K} e^{I J} \otimes e_{K}$. The map $\hat{f}$ is injective because $f\left(\Lambda^{2} T_{n}^{*} \otimes T_{n}\right) \cap \partial\left(T_{n+m}^{*} \otimes \mathfrak{g l}(n+m)\right)=\{0\}$.

For $i \neq 4,8$, let $\tau_{i}$ denote an element of $W_{i}\left(T_{n}\right)$. It is obvious that $\Lambda^{3} V_{n}^{*} \subset$ $\Lambda^{3} V_{n+m}^{*}$; hence, $\hat{f}\left(\tau_{1}\right) \in W_{1}\left(T_{n+m}\right)$. Then the inclusion easily follows for $\tau_{2}$ : in fact, $\hat{f}\left(\tau_{1}+\tau_{2}\right) \in V_{n+m}^{*} \otimes \Lambda^{2} V_{n+m}^{*}$ and $\hat{f}\left(\tau_{2}\right)$ has no component in $\Lambda^{3} V_{n+m}^{*}$ because $\tau_{2}$ has no component in $\Lambda^{3} V_{n}^{*}$. Now recall that $W_{3}\left(T_{n}\right)$ is the kernel of the contraction $V_{n}^{*} \otimes \Lambda^{2} V_{n} \rightarrow V_{n}$; it easily follows that $\hat{f}\left(\tau_{3}\right)$ belongs to the kernel of the corresponding contraction defined on $V_{n+m}^{*} \otimes \Lambda^{2} V_{n+m}$.

Finally, if $\tau_{4}=a_{i} e^{k} \otimes e_{k i}$, then $\hat{f}\left(\tau_{4}\right) \in V_{n+m}^{*} \otimes \Lambda^{2} V_{n+m}$ is given by

$\hat{f}\left(\tau_{4}\right)=a_{i} e^{k} \otimes e_{k i}=\left(a_{i} e^{k} \otimes e_{k i}-\frac{n-1}{m} a_{i} f^{h} \otimes f_{h} \wedge e_{i}\right)+\frac{n-1}{m} a_{i} f^{h} \otimes f_{h} \wedge e_{i}$,

where the first summand lies in $W_{3}\left(T_{n+m}\right)$ and the second one lies in $W_{4}\left(T_{n+m}\right)$; a similar decomposition applies to any element $\tau_{4}^{\prime}=a_{i}^{\prime} f^{k} \otimes f_{k i}$ of $W_{4}\left(T_{n+m}\right)$. Writing explicitly the image in $W_{3}\left(T_{n+m}\right)$ of an element $\tau_{3}+\tau_{4}+\tau_{3}^{\prime}+\tau_{4}^{\prime}$ and imposing that the component in $V_{m}^{*} \otimes\left(V_{m} \wedge V_{n}\right)$ is zero we obtain $\tau_{4}=0$; similarly, one finds that $\tau_{4}^{\prime}=0$. Injectivity follows.

The same arguments can be applied to $\tau_{5}, \tau_{6}, \tau_{7}$ and $\tau_{8}$.

At the geometric level, given two almost parahermitian manifolds $\left(N, g_{n}\right.$, $\left.K_{n}, F_{n}\right)$ and $\left(M, g_{m}, K_{m}, F_{m}\right)$, on the product $N \times M$ we consider the natural almost parahermitian structure

$$
\left(N \times M, g_{n}+g_{m}, K_{n}+K_{m}, F_{n}+F_{m}\right) .
$$


In our setting, an intrinsic torsion class is a subset of $\left\{\mathcal{W}_{1}, \ldots, \mathcal{W}_{8}\right\}$; it is customary to represent a subset $\left\{\mathcal{W}_{i_{1}}, \ldots, \mathcal{W}_{i_{h}}\right\}$ as a formal sum

$$
\mathcal{W}_{i_{1}}+\cdots+\mathcal{W}_{i_{h}} .
$$

Accordingly, the union of two intrinsic torsion classes $I$ and $J$ is written as $I+J$.

An almost parahermitian manifold $(M, g, K, F)$ is in the intrinsic torsion class (19) if the components of the intrinsic torsion which are not identically zero are precisely $\tau_{i_{1}}, \ldots, \tau_{i_{h}}$; we write $\mathcal{W}(M)=\mathcal{W}_{i_{1}}+\cdots+\mathcal{W}_{i_{h}}$.

Proposition 37. If $(N, g, K, F)$ is a $2 n$-dimensional almost parahermitian manifold with $\tau_{4}=0=\tau_{8}$, then for each $m \in \mathbb{N}$ the natural almost parahermitian structure on the $2(m+n)$-dimensional manifold $N \times \mathbb{R}^{2 m}$ is in the same intrinsic torsion class.

Proof. Follows from Proposition 12 and Lemma 36

More generally, we can find non-trivial intrinsic torsion classes by combining two almost parahermitian manifolds. Indeed, by the same arguments we can prove the following:

Proposition 38. Let $M, N$ be two almost parahermitian manifolds of dimension respectively $2 m$ and $2 n$, each with $\tau_{4}=0=\tau_{8}$; then the intrinsic torsion class of the natural almost parahermitian structure on $M \times N$ is the union of the intrinsic torsion classes of $M$ and $N$.

The general case requires more notation. We say a map from the set of intrinsic torsion classes to itself is additive if it satisfies $g(I+J)=g(I)+g(J)$. Consider the additive map

$$
g: \mathcal{W}_{i} \rightarrow \begin{cases}\mathcal{W}_{i} & i \neq 4,8 \\ \mathcal{W}_{3}+\mathcal{W}_{4} & i=4 \\ \mathcal{W}_{7}+\mathcal{W}_{8} & i=8\end{cases}
$$

again by Lemma 36, we find:

Proposition 39. The intrinsic torsion of the natural almost parahermitian structure on a product $N \times M$ is

$$
\mathcal{W}(N \times M)=g(\mathcal{W}(N)+\mathcal{W}(M)) .
$$

In the situation of Proposition 39, if $M$ and $N$ are Einstein with the same scalar curvature $s$, then the product $M \times N$ is also Einstein with scalar curvature $s$. In particular, the results of Section 7 imply:

Proposition 40. Each intrinsic torsion class involving only $\mathcal{W}_{1}, \mathcal{W}_{2}, \mathcal{W}_{3}, \mathcal{W}_{5}$, $\mathcal{W}_{6}$ and $\mathcal{W}_{7}$ contains compact manifolds with a Ricci-flat $\mathrm{GL}(n, \mathbb{R})$-structure for $n \gg 1$.

Unlike $\mathcal{W}_{1}$ and $\mathcal{W}_{2}$, the intrinsic torsion classes $\mathcal{W}_{3}$ and $\mathcal{W}_{4}$ are not Ricci-flat; this is indicated by the formulae of Theorem 15, and can be verified through the following examples: 
Example 41. In the class $\mathcal{W}_{3}$, the standard $\operatorname{SL}(3, \mathbb{R})$-structure on the Lie algebra $(0,0,0,0,45,46)$ has intrinsic torsion

$$
\tau_{3}=\frac{1}{2} e_{2} \otimes e^{45}-\frac{1}{2} e_{3} \otimes e^{46}
$$

the Ricci tensor is $-e^{4} \otimes e^{4}$.

Example 42. A non-Einstein example with intrinsic torsion contained in $W_{4}$ is the standard $\mathrm{SL}(3, \mathbb{R})$-structure on the Lie algebra

$$
(-14,0,0,0,45,46)
$$

In this case the Ricci curvature is $e^{4} \otimes e^{4} \in S^{2} V$.

Remark 8. Using this last example, together with those of Section 7 the construction of Proposition 39 allows one to produce almost parahermitian structures in any given intrinsic torsion class.

\section{References}

[1] D. V. Alekseevsky and B. N. Kimel'fel'd. Structure of homogeneous Riemannian spaces with zero Ricci curvature. Funkcional. Anal. i PriloŽen., $9(2): 5-11,1975$.

[2] D. V. Alekseevsky, C. Medori, and A. Tomassini. Homogeneous paraKählerian Einstein manifolds. Uspekhi Mat. Nauk, 64(1(385)):3-50, 2009.

[3] D. Angella and F. A. Rossi. Cohomology of D-complex manifolds. Differential Geom. Appl., 30(5):530-547, 2012.

[4] T. Aubin. Équations du type Monge-Ampère sur les variétés kählériennes compactes. Bull. Sci. Math. (2), 102(1):63-95, 1978.

[5] L. Bedulli and L. Vezzoni. The Ricci tensor of SU(3)-manifolds. J. Geom. Phys., 57(4):1125-1146, 2007.

[6] R. L. Bryant. Metrics with exceptional holonomy. Annals of Mathematics, 126:525-576, 1987.

[7] R. L. Bryant. Some remarks on $G_{2}$-structures. In Proceedings of Gökova Geometry-Topology Conference 2005, pages 75-109. Gökova Geometry/Topology Conference (GGT), Gökova, 2006.

[8] S. Chiossi and S. Salamon. The intrinsic torsion of $S U(3)$ and $G_{2}$ structures. In Differential Geometry, Valencia 2001, pages 115-133. World Scientific, 2002.

[9] M. Chursin, L. Schäfer, and K. Smoczyk. Mean curvature flow of spacelike Lagrangian submanifolds in almost para-Kähler manifolds. Calc. Var. Partial Differential Equations, 41(1-2):111-125, 2011.

[10] D. Conti. Intrinsic torsion in quaternionic contact geometry. Ann. Sc. Norm. Super. Pisa Cl. Sci. (5), 16(2):625-674, 2016. 
[11] V. Cortés, T. Leistner, L. Schäfer, and F. Schulte-Hengesbach. Half-flat structures and special holonomy. Proc. Lond. Math. Soc. (3), 102(1):113158, 2011.

[12] V. Cortés, C. Mayer, T. Mohaupt, and F. Saueressig. Special geometry of Euclidean supersymmetry. I. Vector multiplets. J. High Energy Phys., 2004(3):028, 73 pp. (electronic), 2004.

[13] V. Cortés and L. Schäfer. Geometric structures on Lie groups with flat bi-invariant metric. J. Lie Theory, 19(2):423-437, 2009.

[14] V. Cruceanu, P. Fortuny, and P. M. Gadea. A survey on paracomplex geometry. Rocky Mountain J. Math., 26(1):83-115, 1996.

[15] F. Etayo, R. Santamaría, and U. R. Trías. The geometry of a bi-Lagrangian manifold. Differential Geom. Appl., 24(1):33-59, 2006.

[16] W. Fulton and J. Harris. Representation theory, volume 129 of Graduate Texts in Mathematics. Springer-Verlag, New York, 1991.

[17] P. M. Gadea and J. M. Masque. Classification of almost para-Hermitian manifolds. Rend. Mat. Appl. (7), 11(2):377-396, 1991.

[18] M.-P. Gong. Classification of nilpotent Lie algebras of dimension 7 (over algebraically closed fields and R). ProQuest LLC, Ann Arbor, MI, 1998. Thesis (Ph.D.)-University of Waterloo (Canada).

[19] A. Gray and L. Hervella. The sixteen classes of almost Hermitian manifolds. Ann. Mat. Pura e Appl., 282:1-21, 1980.

[20] F. R. Harvey and Jr. H. B. Lawson. Split special Lagrangian geometry. In Metric and differential geometry, volume 297 of Progr. Math., pages 43-89. Birkhäuser/Springer, Basel, 2012.

[21] S. Ivanov and S. Zamkovoy. Parahermitian and paraquaternionic manifolds. Differential Geom. Appl., 23(2):205-234, 2005.

[22] Y.-H. Kim, R. J. McCann, and M. Warren. Pseudo-Riemannian geometry calibrates optimal transportation. Math. Res. Lett., 17(6):1183-1197, 2010.

[23] P. Libermann. Sur les variétés presque paracomplexes. In Colloque de topologie et géométrie différentielle, Strasbourg, 1952, no. 5, page 10. La Bibliothèque Nationale et Universitaire de Strasbourg, 1953.

[24] F. Martín Cabrera and A. Swann. Curvature of almost quaternionHermitian manifolds. Forum Math., 22(1):21-52, 2010.

[25] Y. Matsushita. Counterexamples of compact type to the Goldberg conjecture and various version of the conjecture. In Topics in contemporary differential geometry, complex analysis and mathematical physics, pages 222-233. World Sci. Publ., Hackensack, NJ, 2007.

[26] F. A. Rossi. D-complex structures: cohomological properties and deformations. PhD thesis, Università degli Studi di Milano - Bicocca, 2013. http://hdl. handle.net/10281/41976. 
[27] L. Schäfer. Conical Ricci-flat nearly para-Kähler manifolds. Ann. Global Anal. Geom., 45(1):11-24, 2014.

[28] L. Schäfer and F. Schulte-Hengesbach. Nearly pseudo-Kähler and nearly para-Kähler six-manifolds. In Handbook of pseudo-Riemannian geometry and supersymmetry, volume 16 of IRMA Lect. Math. Theor. Phys., pages 425-453. Eur. Math. Soc., Zürich, 2010.

[29] K. Sekigawa. On some compact Einstein almost Kähler manifolds. J. Math. Soc. Japan, 39(4):677-684, 1987.

[30] N. Steenrod. The Topology of Fibre Bundles. Princeton Mathematical Series, vol. 14. Princeton University Press, Princeton, N. J., 1951. Reprinted in Princeton Landmarks in Mathematics, Princeton University Press, Princeton, NJ, (1999).

[31] S.-T. Yau. On the Ricci curvature of a compact Kähler manifold and the complex Monge-Ampère equation. I. Comm. Pure Appl. Math., 31(3):339411, 1978.

Dipartimento di Matematica e Applicazioni, Università di Milano Bicocca, via Cozzi 55, 20125 Milano, Italy.

diego.conti@unimib.it

federico.rossi@unimib.it 